\title{
The Boundary Layer Dynamics of Tropical Cyclone Rainbands
}

\author{
JEFFREY D. KEPERT \\ Bureau of Meteorology, Melbourne, Victoria, Australia
}

(Manuscript received 11 May 2018, in final form 2 August 2018)

\begin{abstract}
Spiral bands are ubiquitous features in tropical cyclones and significantly affect boundary layer thermodynamics, yet knowledge of their boundary layer dynamics is lacking. Prompted by recent work that has shown that relatively weak axisymmetric vorticity perturbations outside of the radius of maximum winds in tropical cyclones can produce remarkably strong frictional convergence, and by the observation that most secondary eyewalls appear to form by the "wrapping up" of a spiral rainband, the effect of asymmetric vorticity features that mimic spiral bands is studied. The mass field corresponding to an axisymmetric vortex with added spiral vorticity band is constructed using the nonlinear balance equation, and supplied to a three-dimensional boundary layer model. The resulting flow has strong low-level convergence and a marked updraft extending along the vorticity band and some distance downwind. There is a marked along-band wind maximum in the upper boundary layer, similar to observations, which is up to about $20 \%$ stronger than the balanced flow. A marked gradient in the inflow-layer depth exists across the band and there is an increase in the surface wind factor (the ratio of surface wind speed to nonlinear-balanced wind speed) near the band. The boundary layer dynamics near a rainband therefore form a continuum with the flow near a secondary eyewall. None of these features are due to convective momentum transports, which are absent from the model. The sensitivities of the flow to band length, width, location, crossing angle, and amplitude are examined, and the possible contribution of boundary layer dynamics to the formation of the tropical cyclone rainbands discussed.
\end{abstract}

\section{Introduction}

In the 1940s, the first radar images of tropical cyclones revealed what was then a surprising fact: much of the rainfall that surrounds the nearly circular eye is organized into spiral bands (Maynard 1945; Wexler 1947). Subsequent work has revealed that these bands have a wide range of spatial scales, may either propagate relative to the storm or be stationary, and consist of a mix of stratiform and convective precipitation. Major bands are typically mainly cumuliform at the upwind end, tending toward stratiform downwind, although individual bands can exhibit considerable spatial and temporal variability (Barnes et al. 1983; Didlake and Houze 2013b). Although we have developed a good observational understanding and classification of the characteristics of spiral bands, our understanding of their dynamics is incomplete. Some studies find that lowerwavenumber propagating bands are well modeled as vortex Rossby waves (Reasor et al. 2000; Corbosiero

\footnotetext{
Corresponding author: Jeffrey D. Kepert, jeff.kepert@bom. gov.au
}

et al. 2006) while others differ (Moon and Nolan 2015b). However, there is as yet no satisfactory explanation for major features such as the principle band (Houze 2010).

The dynamics of the tropical cyclone boundary layer have likewise been much studied. One reason for this interest is that it is where much of the impact on humanity occurs, either directly through the winds, or less directly through the transfer of momentum into the ocean, generating currents, waves, and storm surge. A second reason is the boundary layer's central role in tropical cyclone energetics, since the air-sea fluxes are both a major source (latent and sensible heat) and sink (surface drag) of energy to the storm. A third reason is that the frictional convergence in the tropical cyclone boundary layer strongly influences the location and strength of the convection near primary and secondary eyewalls and, hence, contributes to their development (Kepert 2013; Kepert and Nolan 2014; Zhang et al. 2017). Thus, the boundary layer plays an essential role in theories of tropical cyclone intensification (Ooyama 1969) and potential intensity (Emanuel 1986).

Several observational studies of the boundary layer flow near a rainband have revealed a reasonably consistent 
picture. The band often forms a partial barrier to inflow, with markedly stronger and deeper inflow, a greater inflow angle, and higher $\theta_{e}$ on the outside of the band than on the inside. There may be a layer of outflow on top of the inflow. There is often an along-band jet in the lower troposphere and a marked pressure perturbation across the band (Barnes et al. 1983; Barnes and Powell 1995). Barnes and Powell (1995) further noted that the vertical shear below $1 \mathrm{~km}$ is much stronger on the outside of the band than on the inside, which together with the inflow strength and depth differences leads to very different boundary layer wind profiles on either side of the band. Similar features have been reported in numerical simulations. For example, Li and Wang (2012) report an alongband jet at the top of the boundary layer, and Moon and Nolan (2015a) found a decrease in the inflow strength across both principal and secondary rainbands, along with enhanced tangential flow on their outward sides.

One branch of research into the tropical cyclone boundary layer has been through diagnostic models of the flow. Such models aim to diagnose the flow within the boundary layer, given a pressure field that represents the rest of the cyclone. There is a wide variety of such models, which can be classified according to the additional assumptions (such as axisymmetry, depth averaging, or linearization) made to obtain a solution (Kepert 2010). The more sophisticated of such models are well able to reproduce the flow in full-physics models (Kepert and Nolan 2014; Zhang et al. 2017) and observations (Kepert 2006a,b; Schwendike and Kepert 2008). Boundary layer models have been used to understand certain important observed features of the tropical cyclone boundary layer, including the supergradient flow in the upper part of the inflow layer, and the higher values of the surface wind factor near and within the RMW than at larger radii (Kepert 2001; Kepert and Wang 2001; Powell et al. 2009).

These models aim to diagnose one side of what is in reality a two-way interaction: the boundary layer flow depends on the pressure pattern imposed by the rest of the cyclone, but the boundary layer is unable to modify that pressure field as it would in reality, because that pressure field is imposed and held fixed as an input to the model. One important way that the boundary layer can feed back to the cyclone as a whole is through the distribution and strength of frictional convergence, for frictionally forced ascent (along with the thermodynamic environment) influences the distribution and strength of convection and, hence, latent heat release within the vortex. However, the presence of horizontal convergence within the boundary layer beneath moist convection does not necessarily imply that the convergence is caused by friction, for buoyant ascent within cumuliform clouds can also cause near-surface convergence (Raymond and Herman 2012). Methods to estimate the frictional convergence are therefore needed, which these models provide.

The linearized expression for the frictionally forced vertical motion in an axisymmetric vortex is

$$
w_{\infty}=\frac{1}{r} \frac{\partial}{\partial r} \frac{r C_{d} v_{\mathrm{gr}}\left(v_{\mathrm{gr}}+2 v^{\prime}\right)}{\zeta_{\mathrm{gr}}+f},
$$

where $w_{\infty}$ is the frictional updraft at the top of the boundary layer, $r$ is the radius, $C_{d}$ is the drag coefficient, $v_{\mathrm{gr}}$ is the gradient wind and $\zeta_{\mathrm{gr}}$ its vorticity, and $v^{\prime}$ is the departure of the surface azimuthal flow from $v_{\text {gr }}$ (Kepert 2001). Recent work (Kepert 2013) has related this formula to the classical expression for Ekman pumping. In particular, (1) is equivalent, apart from the linearization of the surface boundary condition, to

$$
w_{\infty}=\mathbf{k} \cdot \nabla \times \frac{\boldsymbol{\tau}}{\rho\left(\zeta_{\mathrm{gr}}+f\right)},
$$

where $\tau$ is the surface stress and $\rho$ is the air density, which differs from the classical Ekman expression only in that the Coriolis parameter $f$ is moved inside the curl operator and replaced by the absolute vorticity $\zeta_{\mathrm{gr}}+f$. A similar equivalence applies for Ooyama's (1969) similar expression. If the radial derivative in (1) is expanded,

$$
\begin{aligned}
w_{\infty}= & -\frac{1}{\left(\zeta_{\mathrm{gr}}+f\right)^{2}} \frac{\partial \zeta_{\mathrm{gr}}}{\partial r} C_{d} v_{\mathrm{gr}}\left(v_{\mathrm{gr}}+2 v^{\prime}\right) \\
& +\frac{1}{r\left(\zeta_{\mathrm{gr}}+f\right)} \frac{\partial\left[r C_{d} v_{\mathrm{gr}}\left(v_{\mathrm{gr}}+2 v^{\prime}\right)\right]}{\partial r}
\end{aligned}
$$

Kepert (2013) further showed that near eyewalls, the first term, which he calls the radial derivative term, dominates over the second term, which contains the curl of the stress. Hence, the fact that the vortex vorticity is inside the curl operators in (1) and (2) leads to a distinctly different physical interpretation to that in classical Ekman theory. The factor $\left(\zeta_{\mathrm{gr}}+f\right)^{-2}$ in (3) is important, for it allows quite weak vorticity gradients to produce significant updrafts, provided that they occur in an environment of low vorticity; that is, away from the primary radius of maximum winds (RMW) and eye (Kepert 2013). This sensitivity has important implications for secondary eyewall formation, summarized below.

Nonlinearity in the tropical cyclone boundary layer, particularly the vertical advection and that part of the radial advection removed in the linearization, modifies the flow from the linear solution. First, the eyewall updraft(s) are displaced inward by a distance that scales as $-u_{10} / I$, where $-u_{10}$ is the inflow at $10 \mathrm{~m}$ and $I$ is the 
inertial stability of the gradient wind. Eyewall updrafts occur near a marked inward increase in vorticity. This updraft, which occurs near the outer edge of the steplike inward vorticity increase in the linearized boundary layer model, is displaced inward to be at or inside the top of the step in the nonlinear model (Kepert 2017). Second, (1) is very sensitive to small-scale features in the vorticity, but the nonlinearity filters out this response (Kepert 2017). A third important difference is that the nonlinear model develops marked supergradient flow in the upper part of the inflow layer, and that this supergradient flow is much weaker in the linearized model. While some studies (e.g., Huang et al. 2012; Abarca and Montgomery 2013) have attributed the frictional convergence to the outward acceleration associated with this imbalance, Kepert and Nolan (2014) argue that that attribution is incorrect, and that both the strongly supergradient flow and the updraft are together caused by the locally increased vorticity. These different views are summarized in Table 1 in Kepert and Nolan (2014) and are further discussed in Kepert (2017).

Almost all the diagnostic models of the tropical cyclone boundary layer reviewed in Kepert (2010) assume an axisymmetric pressure field, the only exceptions being two single-column models that incorporate no vortex structure at all. The only source of boundary layer asymmetry investigated using these diagnostic models has been asymmetric friction, due either to storm motion (Shapiro 1983; Kepert 2001; Kepert and Wang 2001) or to proximity to land (Kepert 2002a,b, 2006b; May et al. 2008; Ramsay et al. 2009). The effects of asymmetries in the pressure field itself have not been studied.

Yet, while the real tropical cyclone pressure field is nearly axisymmetric, it is not exactly so. Large-scale sources of asymmetry include Earth's curvature, leading to the formation of beta gyres (Chan and Kepert 2010, chapter 4 ), and the effects of the storm environment, including vertical wind shear (Chan and Kepert 2010, chapter 1). At smaller scales, individual convective cells make small-scale perturbations to the vorticity, which will be reflected to some degree in the pressure field. Intermediate to these scales, spiral bands are often associated with wind maxima (Samsury and Zipser 1995; Houze 2010), and persist for long enough that the wind maximum can be expected to coexist with a balanced asymmetric pressure signal. The principle aim of this study is to explore the effect of asymmetric pressure features on the boundary layer flow, in particular those caused by spiral rainbands. We will discuss these effects in terms of features that have been previously studied in axisymmetric vortices, including the location and strength of the frictional updraft, the strength of the surface wind relative to that above the boundary layer, the angle by which the wind direction changes through the depth of the boundary layer, and the extent and heights at which flow in the upper boundary layer is stronger than the balanced flow.

Our interest in the boundary layer flow in spiral rainbands is particularly motivated by recent work on the role of the boundary layer in eyewall replacement cycles (Kepert 2013; Kepert and Nolan 2014). Their discovery of the high sensitivity of the frictional updraft to the addition of small, axisymmetric vorticity bumps raises the following question: what is the influence of vorticity features of other shapes? A further motivation comes from the observation that much of the existing theoretical work on mechanisms of secondary eyewall formation is essentially axisymmetric. While the vortex Rossby wave theory of Montgomery and Kallenbach (1997), which discusses the symmetric response to momentum accumulation from asymmetric waves, and the beta-skirt axisymmetrization theory of Terwey and Montgomery (2008), which discusses the axisymmetrization of convectively generated vorticity perturbations, are partial exceptions, they nevertheless explore the symmetric response to asymmetric processes. In contrast to the axisymmetric theories, examination of sequences of satellite images shows that the majority of secondary eyewalls appear to form via the "wrapping up" of a principal rainband. It is unclear how this observed evolution relates to the body of secondary eyewall theory, although Didlake et al. (2018) have recently proposed that a mesoscale descending inflow jet in the stratiform sector may trigger a convective updraft and accelerate the tangential winds there. Within the context of recent work on the boundary layer contribution to secondary eyewall formation (Kepert 2013; Kepert and Nolan 2014), we therefore seek to examine the question of how the boundary layer flow beneath a spiral band of convection compares to that beneath a concentric ring, and how this flow changes if the spiral band wraps up into a secondary eyewall.

\section{Modeling the boundary layer flow near a spiral rainband}

To model the boundary layer flow near a spiral rainband, we adapt the model introduced by Kepert and Wang (2001) and subsequently modified by Kepert (2012) to allow the use of an asymmetric forcing pressure field. After first describing how to parameterize the pressure signal of spiral bands in tropical cyclones, we then discuss the necessary model modifications.

\section{a. Parameterized spiral bands}

We construct a balanced, asymmetric pressure field to represent a tropical cyclone with one or more stationary 
rainbands as follows. The symmetric cyclone is modeled as a modified Rankine vortex, written in terms of vorticity and smoothed in the vicinity of the RMW as in Kepert (2013):

$$
\zeta_{\mathrm{tc}}(r)=w(r) \zeta_{\mathrm{eye}}+[1-w(r)] \frac{1-\alpha}{2} \zeta_{\mathrm{eye}}\left(\frac{r}{r_{t}}\right)^{-1-\alpha},
$$

where $\zeta_{\text {eye }}$ is the vorticity within the eye, $\alpha$ is the decay coefficient, and $w(r)$ is a smooth function that decreases from 1 to 0 over some distance surrounding the radius $r_{t}$ of transition from solid-body rotation in the eye to a power-law decay of wind and vorticity outside of it. In all the cases presented here, the base vortex has a core vorticity of $\zeta_{\text {eye }}=4 \times 10^{-3} \mathrm{~s}^{-1}$, a transition radius of $r_{t}=25 \mathrm{~km}$, a decay coefficient of $\alpha=0.5$, and smoothing at the transition radius over $10 \mathrm{~km}$. The wind field can be recovered from the vorticity by a radial integration. The resulting cyclone has a maximum wind speed of $47 \mathrm{~m} \mathrm{~s}^{-1}$ at radius $27 \mathrm{~km}$.

The rainband is modeled as a spiral band of vorticity of the form

$$
\zeta_{\mathrm{rb}}=\zeta_{0} \exp \left(-\frac{d^{2}}{2 L^{2}}\right)
$$

where $d$ is the distance from the axis of the band, $L$ is a length scale, and $\zeta_{0}$ is the amplitude of the band. The axis of the band is modeled as a logarithmic spiral,

$$
r=a \exp (b \lambda),
$$

for the chosen range of azimuth $\lambda$. Logarithmic spirals have the property that the angle at which the spiral crosses circles centered on the origin is constant. The spirals are defined by their crossing angle $\theta$, angular length $\Lambda$, and radius of the midpoint $r_{\mathrm{sp}}$, which together determine $b=\tan \theta$ and $a=r_{\mathrm{sp}} \exp (-b \Lambda / 2)$. Together with the width and amplitude parameters in (5), the spiral parameter space is five-dimensional.

We justify modeling rainbands as bands of vorticity on the basis of both observations and theory. Samsury and Zipser (1995) analyzed a substantial amount of aircraft radial leg data in hurricanes; while the data were at various flight levels, more than half were at $850 \mathrm{hPa}$. They found that about $20 \%$ of radial legs had secondary wind maxima meeting their criteria, and that most of these secondary wind maxima had collocated rainbands; these wind maxima imply local perturbations to the vorticity field. Although over $70 \%$ of rainbands in Samsury and Zipser (1995) did not have secondary wind maxima, it is possible that these rainbands simply had weaker vorticity features, too weak to be associated with a local wind maximum. ${ }^{1}$ Powell (1990) presented a Doppler radar-based observational study of a rainband, which showed clear wind and vorticity maxima aligned along the band axis. Barnes and Powell (1995) similarly show a substantial tangential velocity maximum outside of a rainband in Hurricane Gilbert. Judt and Chen (2010) present Doppler radar analyses of vorticity showing clear vorticity maxima in the rainbands, similar to those in their high-resolution model simulations. Didlake and Houze (2009) analyzed Doppler radar data of the principal rainband of Hurricane Katrina (2005), and describe a marked low-level convective-scale azimuthal wind maximum associated with the inner-edge downdraft of that band, while Didlake and Houze (2013a) report a similar structure in the upwind end of the principal rainband in Hurricane Rita (2005). In contrast, Didlake and Houze (2013b) note a midlevel, rather than a low-level, azimuthal wind maximum in their study of the downwind, stratiform sector of the principal rainband in Hurricane Rita (2005).

The observations of low-level azimuthal wind maxima in the convective part of the band are consistent with theory, for buoyant convection can spin up existing vertical vorticity through vortex tube stretching, as well as tilting horizontal vorticity into the vertical. On the other hand, the stratiform sector of the rainband has relatively weak, decaying, convective cells, which would not stretch low-level vorticity to the same degree, although the stratiform heating profile may generate midlevel convergence, thereby altering the vorticity (May and Holland 1999; Franklin et al. 2006). Overall, although theory and observations do not paint an entirely consistent picture, it is reasonable to assume that a spiral rainband causes a local region of increased vorticity of broadly similar shape.

Given the vorticity of the rainband and tropical cyclone, we need to find the corresponding velocity and mass fields. We assume the flow above the boundary layer to be horizontally nondivergent, so we represent the horizontal velocity field by a streamfunction $\psi$ and solve

$$
\nabla^{2} \psi=\zeta
$$

The only challenge here is an appropriate boundary condition for a numerical solution, so as to not introduce artifacts of the finite domain to the solution. If $\zeta$ is axisymmetric, then solving (7) reduces to a radial

\footnotetext{
${ }^{1}$ In support of this possibility, several studies of secondary eyewall formation show that the azimuthal-mean vorticity or potential vorticity maximum forms before the azimuthal-mean tangential wind maximum (Judt and Chen 2010; Abarca and Corbosiero 2011; Bell et al. 2012; Wu et al. 2012).
} 
integration. Hence, we solve the tropical cyclone (tc, assumed axisymmetric) and rainband ( $\mathrm{rb}$ ) parts of the flow separately:

$$
\begin{aligned}
& \nabla^{2} \psi_{\mathrm{tc}}=\zeta_{\mathrm{tc}} \text { and } \\
& \nabla^{2} \psi_{\mathrm{rb}}=\zeta_{\mathrm{rb}},
\end{aligned}
$$

where solving (8) requires a radial integral, and the smaller amplitude of $\zeta_{\mathrm{rb}}$ reduces the boundary condition artifacts in the solution of (9). We solve (9) for $\psi_{\mathrm{rb}}$ using an algebraic multigrid solver (Olson and Schroder 2018). This solver is very efficient for large problems, so we can further reduce the risk of contamination from the boundary condition by making the domain much larger than will be used in the boundary layer model. We found that solving on a $1001 \times 1001$ gridpoint square domain with $3-\mathrm{km}$ grid spacing and the boundary condition $\psi=0$, and using only the middle $201 \times 201$ square, led to only a very low azimuthal wavenumber- 4 signal in the central region, deemed to be sufficiently free of artifacts from the square boundary. On this domain, the solver takes only a few seconds to converge on a desktop computer.

We will see in the next section that the balanced mass field is not strictly needed. Nevertheless, it is interesting, so we now show how to calculate it. The nonlinear balance equation (NLBE) can be written

$$
\nabla^{2} \phi=2\left(\psi_{x x} \psi_{y y}-\psi_{x y}^{2}\right)+f \nabla^{2} \psi
$$

where $\phi$ is the geopotential, $\psi$ the streamfunction, and subscripts $x$ and $y$ denote derivatives. The axisymmetric geopotential $\phi_{\mathrm{tc}}$ corresponding to $\zeta_{\mathrm{tc}}$ is found using the gradient wind equation and, therefore, also satisfies the NLBE:

$$
\nabla^{2} \phi_{\mathrm{tc}}=2\left(\psi_{\mathrm{tc} x x} \psi_{\mathrm{tc} y y}-\psi_{\mathrm{tc} x y}^{2}\right)+f \nabla^{2} \psi_{\mathrm{tc}}
$$

Substituting $\psi=\psi_{\mathrm{tc}}+\psi_{\mathrm{rb}}$ and $\phi=\phi_{\mathrm{tc}}+\phi_{\mathrm{rb}}$ into the NLBE and using (11), we obtain an equation for the part of the geopotential due to the rainband:

$$
\begin{aligned}
\nabla^{2} \phi_{\mathrm{rb}}= & 2\left(\psi_{\mathrm{rb} x x} \psi_{\mathrm{tc} y y}+\psi_{\mathrm{tc} x x} \psi_{\mathrm{rb} y y}+\psi_{\mathrm{rb} x x} \psi_{\mathrm{rb} y y}\right. \\
& \left.-2 \psi_{\mathrm{rb} x y} \psi_{\mathrm{tc} x y}-\psi_{\mathrm{rb} x y}^{2}\right)+f \nabla^{2} \psi_{\mathrm{rb}}
\end{aligned}
$$

which can be solved for $\phi_{\mathrm{rb}}$ using the same solver and domain as used for finding $\psi_{\mathrm{rb}}$. Note that $\phi_{\mathrm{rb}}$ depends on both $\zeta_{\mathrm{rb}}$ and $\zeta_{\mathrm{tc}}$. Again, we reduce the boundary condition artifacts by solving only for the smaller $\phi_{\mathrm{rb}}$, rather than the full $\phi$.

Figure 1 shows the results of this procedure for a case in which the spiral is $\Lambda=180^{\circ}$ long, has a crossing angle of $\theta=10^{\circ}$, and a midpoint radius of $r_{\mathrm{sp}}=75 \mathrm{~km}$. There is scant information on band crossing angles in the literature, but Barnes et al. (1983) and Barnes and Powell (1995) note an angle of $9^{\circ}$, while Didlake and Houze (2013b) give an equation that approximates the band they analyzed and implies a crossing angle in the range of about $4^{\circ}-12^{\circ}$. The Gaussian width scale is $L=10 \mathrm{~km}$, and the amplitude $\zeta_{0}=4 \times 10^{-4} \mathrm{~s}^{-1}$ is one-tenth that in the cyclone's eye. The spiral band part of the flow weakens the flow at the RMW on the adjacent side of the storm and strengthens it on the opposite side. However, the asymmetries in $\varphi$ and $\psi$ are more difficult to discern in the full-field plots. The maximum $\left|\nabla_{H} \phi_{\mathrm{rb}}\right|$ is $5.0 \times$ $10^{-3} \mathrm{~m} \mathrm{~s}^{-2}$, while $\left|\nabla_{H} \phi_{\mathrm{tc}}\right|$ at the same point is $1.26 \times$ $10^{-2} \mathrm{~m} \mathrm{~s}^{-2}$. The rainband thus increases the geopotential gradient at this location by almost $40 \%$, similar to the increase observed by Barnes and Powell (1995, their Fig. 5d).

The parameter space explored in this paper is set out in Table 1.

\section{b. Revisions to the tropical cyclone boundary layer model}

As discussed above, we take the forcing pressure field to be the solution to the NLBE for a vorticity structure consisting of a symmetric modified Rankine vortex (smoothed near the RMW) with a spiral band of vorticity. Writing the horizontal velocity vector $\tilde{\mathbf{v}}=\mathbf{v}_{B}+\mathbf{v}$, where $\mathbf{v}_{B}$ is the balanced flow and $\mathbf{v}$ is the departure of the flow from the balanced reference state, the horizontal momentum equation is

$$
\begin{aligned}
\frac{\partial \tilde{\mathbf{v}}}{\partial t}+\tilde{\mathbf{v}} \cdot \nabla_{H} \tilde{\mathbf{v}}+w \frac{\partial \tilde{\mathbf{v}}}{\partial z}+f \mathbf{k} \times \tilde{\mathbf{v}}= & -\nabla_{H} \tilde{\phi}+\frac{\partial}{\partial z} K \frac{\partial \tilde{\mathbf{v}}}{\partial z} \\
& + \text { horiz diff }
\end{aligned}
$$

where $\nabla_{H}$ is the horizontal gradient operator, "horiz diff" is the fourth-order horizontal diffusion, and other symbols have their customary meanings. Because the balanced part of the flow satisfies the NLBE, it must also satisfy the steady frictionless momentum equation:

$$
\mathbf{v}_{B} \cdot \nabla_{H} \mathbf{v}_{B}+f \mathbf{k} \times \mathbf{v}_{B}=-\nabla_{H} \phi_{B} .
$$

Expanding (13) and using (14),

$$
\begin{aligned}
& \frac{\partial \mathbf{v}}{\partial t}+\mathbf{v}_{B} \cdot \nabla_{H} \mathbf{v}+\mathbf{v} \cdot \nabla_{H} \mathbf{v}_{B}+\mathbf{v} \cdot \nabla_{H} \mathbf{v}+w \frac{\partial \mathbf{v}}{\partial z}+f \mathbf{k} \times \mathbf{v} \\
& =\frac{\partial}{\partial z} K \frac{\partial \mathbf{v}}{\partial z}+\text { horiz diff. }
\end{aligned}
$$

This transformation reduces to that in Kepert (2012) when the balanced reference flow is axisymmetric. Note that although we describe how to calculate the mass field in section $2 \mathrm{a}$, we instead incorporate it into the model using (15). 

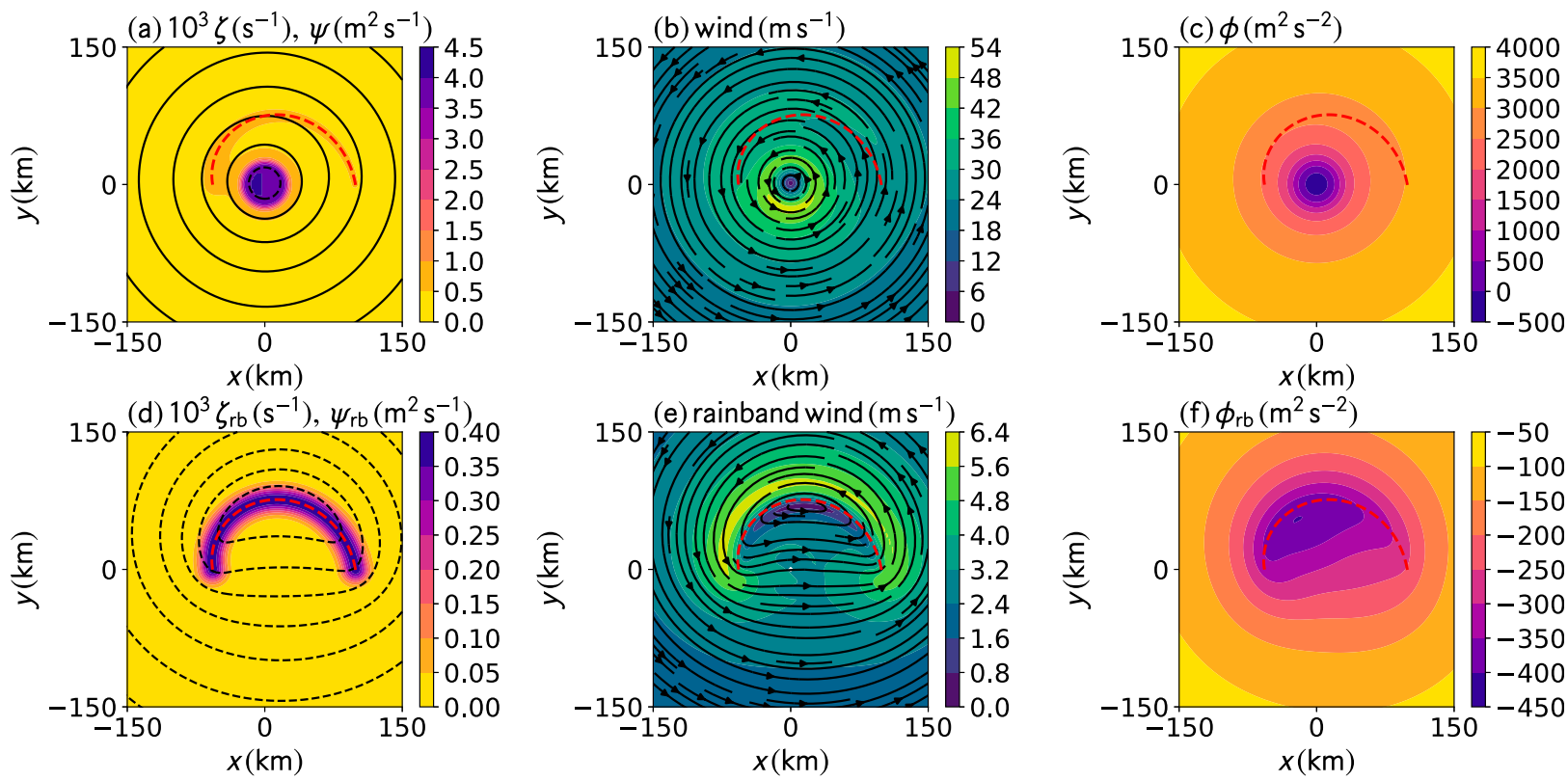

FIG. 1. Parametric balanced tropical cyclone vortex with a rainband: (a) vorticity (shading) and streamfunction (contours; interval $\left.1 \times 10^{6} \mathrm{~m}^{2} \mathrm{~s}^{-1}\right)$, (b) wind speed and streamlines, and (c) geopotential for the full vortex. The red dashed curve marks the axis of the rainband vorticity. (d)-(f) As in (a)-(c), but for just the rainband portion of the flow; the contour interval for the streamfunction is $1 \times 10^{5} \mathrm{~m}^{2} \mathrm{~s}^{-1}$. The tropical cyclone vortex has a core vorticity of $4 \times 10^{-3} \mathrm{~s}^{-1}$ with a transition radius of $25 \mathrm{~km}$ and a decay coefficient of $\alpha=0.5$. The rainband has a vorticity amplitude of $4 \times 10^{-4} \mathrm{~s}^{-1}$, a midpoint radius of $75 \mathrm{~km}$, a crossing angle of $10^{\circ}$, an angular length of $180^{\circ}$, and a scale width of $10 \mathrm{~km}$.

Previous applications of the model have included a thermodynamic budget equation and hydrostatically modified the horizontal pressure gradients in accordance with the thermal field. However, the effect of the temperature budget on these pressure gradients is quite small. Moreover, Kepert et al. (2016) have shown that cloud condensation and rainfall evaporation have a substantial effect on the thermodynamic budget within the tropical cyclone boundary layer. As the boundary layer model is dry, its representation of $\theta$ is therefore somewhat unrealistic, while a more realistic representation would require that we somehow represent the rainfall distribution. Hence, we have removed the hydrostatic adjustment of the horizontal pressure gradients from the model for this study and there is no perturbation pressure gradient term in (15).

Although we will study only stationary storms in this article, we note that only minor modifications are needed for storms moving with a constant translation velocity. In such cases, it is natural to work in a stormfollowing coordinate system. Providing that one adjusts the geopotential field to remove the geostrophic component that balances the coordinate system motion, and that the storm moves with this geostrophic environmental flow, the momentum budget equations are unchanged. Thus, the budget equation for the horizontal divergence, and hence the nonlinear balance equation, are also unchanged. The only change needed is to the surface boundary condition, which needs to account for the motion of Earth's surface in the translating coordinate system.

Vertical turbulent diffusion is parameterized by a first-order closure without stability dependence, the neutral scheme described by Kepert (2012), with the asymptotic mixing length set to $80 \mathrm{~m}$. The surface fluxes are parameterized by a drag coefficient formulation, with the piecewise linear drag coefficient given by

TABLE 1. Parameters used for sensitivity studies. For each row except the last, the nonvaried parameters are $\zeta_{0}=4 \times 10^{-4} \mathrm{~s}^{-1}$, crossing angle $=10^{\circ}$, scale width $L=10 \mathrm{~km}$, azimuthal extent $=180^{\circ}$, and midpoint radius $=75 \mathrm{~km}$. For the last row, the crossing angle is $0^{\circ}$.

\begin{tabular}{lc}
\hline Parameter & Values \\
\hline Band amplitude $\zeta_{0}\left(\mathrm{~s}^{-1}\right)$ & $0,1 \times 10^{-4}, 2 \times 10^{-4}, 4 \times 10^{-4}$, \\
& $8 \times 10^{-4}, 1.6 \times 10^{-3}$ \\
Crossing angle $\theta\left(^{\circ}\right)$ & $0,5,10,15,20,25$ \\
Scale width $L(\mathrm{~km})$ & $5,10,20,40$ \\
Azimuthal extent $\Lambda\left(^{\circ}\right)$ & $0,22.5,45,90,180,360$ \\
Midpoint radius $r_{\mathrm{sp}}$ & $50,75,100,125,150$ \\
$\quad($ spirals $)(\mathrm{km})$ & \\
Radius $($ semicircles $)$ & $50,62,75,87,100,112,125,137,150,162$ \\
$(\mathrm{~km})$ &
\end{tabular}



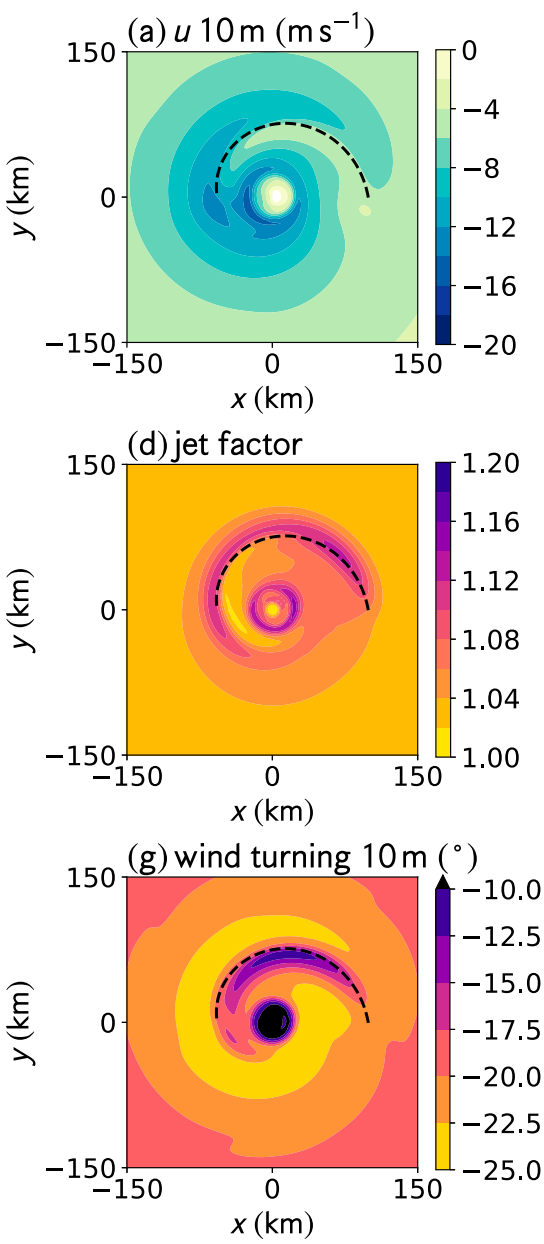

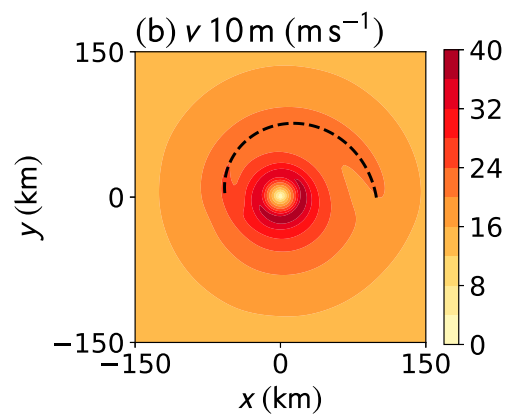

(e) jet height $(\mathrm{m})$
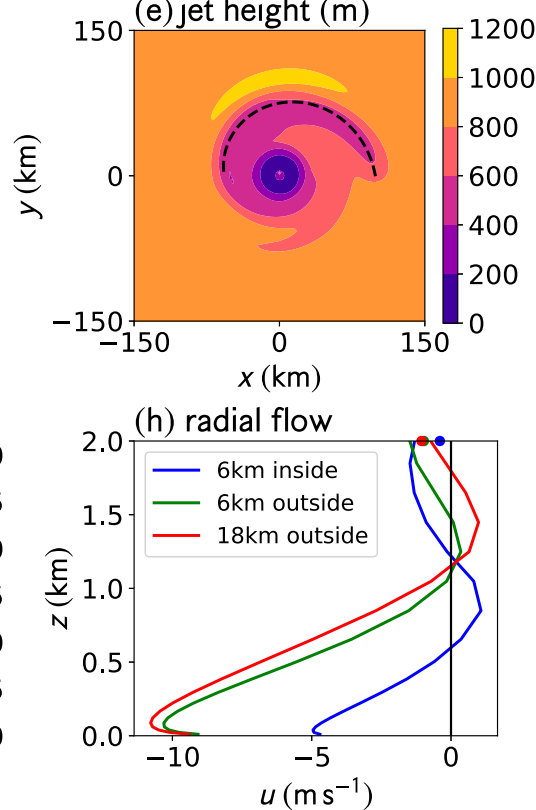
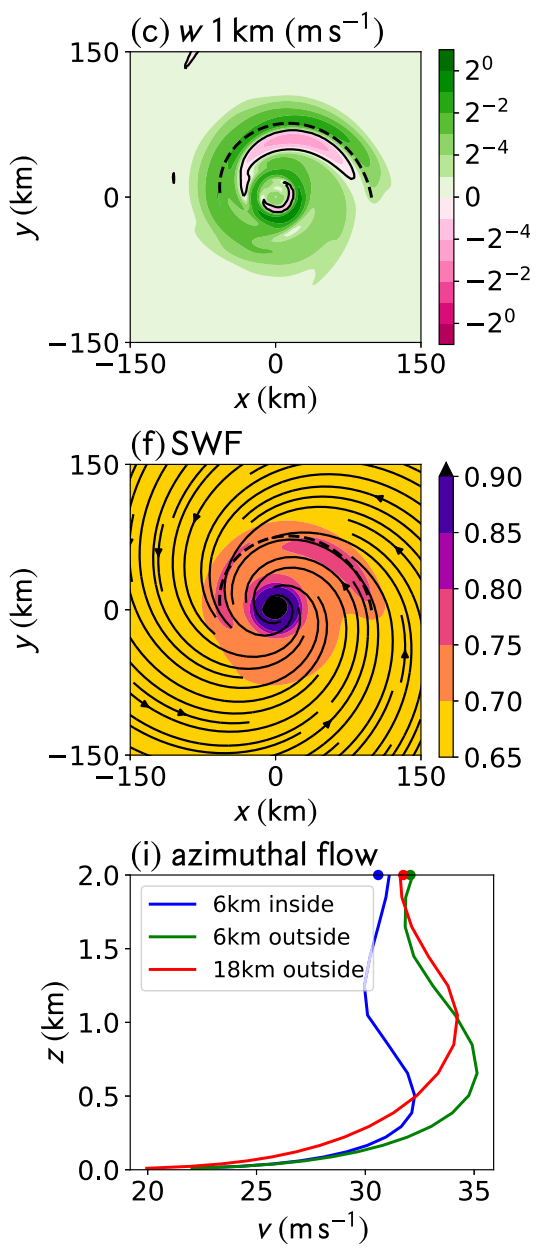

FIG. 2. Simulated boundary layer flow for the vortex in Fig. 1. (a) Radial velocity at $10 \mathrm{~m}$, with a contour interval of $2 \mathrm{~m} \mathrm{~s}^{-1}$; (b) azimuthal velocity at $10 \mathrm{~m}$, with a contour interval of $4 \mathrm{~m} \mathrm{~s}^{-1}$; (c) vertical velocity at $1 \mathrm{~km}$ with the zero contour shown in black and nonzero contours spaced by factors of 2 from $\pm 2^{-5}$ to $\pm 2^{1} \mathrm{~m} \mathrm{~s}^{-1}$; (d) the highest wind speed in the profile, divided by the balanced wind speed in that column, with a contour interval of 0.02 ; (e) the height of the maximum wind speed, with a contour interval of $200 \mathrm{~m}$; (f) the ratio of the 10 - $\mathrm{m}$ wind speed to the balanced wind speed, with a contour interval of 0.05 , together with the streamlines of the $10-\mathrm{m}$ flow; $(\mathrm{g})$ the difference in direction between the $10-\mathrm{m}$ flow and the balanced flow, with turning toward the center of the storm being negative and with a contour interval of $2.5^{\circ}$; (h) vertical profiles of the radial flow $6 \mathrm{~km}$ inside (blue), and 6 (green) and $18 \mathrm{~km}$ (red) outside, of the band axis, at its midpoint, with the balanced radial flow at those points being shown by the like-colored dots; and (i) as in (h), but for the azimuthal flow. Note that the contours in (c) are not linearly spaced but increase by factors of 2 . The black dashed curve in (a)-(g) indicates the axis of the vorticity band.

$$
10^{3} C_{d}= \begin{cases}1.2, & \left|\mathbf{v}_{10}\right|<7.7 \mathrm{~m} \mathrm{~s}^{-1} \\ 0.7+0.065\left|\mathbf{v}_{10}\right|, & 7.7<\left|\mathbf{v}_{10}\right|<24.6 \mathrm{~m} \mathrm{~s}^{-1} \\ 2.3, & 24.6 \mathrm{~m} \mathrm{~s}^{-1}<\left|\mathbf{v}_{10}\right|\end{cases}
$$

\section{Results}

\section{a. Base run}

Figure 2 summarizes the simulated boundary layer flow in the cyclone (with rainband) presented in Fig. 1. There is a band of strong surface winds close to the axis of the vorticity band, with stronger radial inflow outside the band, and weaker inside (Figs. 2a,b). Consistent with these radial flow differences, the turning of the wind direction with height, defined as the difference in the direction of the 10-m and balanced flows, is about $10^{\circ}$ greater on the outside of the band than on the inside (Fig. $2 \mathrm{~g}$ ). The vorticity band thus causes local convergence and confluence maxima, reflected in the marked updraft near the band axis (Fig. 2c). This updraft is slightly outside of the upwind end of the band axis, crosses it about halfway along, and is inside of the downwind end. Although the vorticity in the band is onetenth that in the eye region, the updraft is similar in strength 
to the eyewall updraft. The vorticity band's updraft extends from the downwind end of the band, spiraling inward into the eyewall. A marked region of subsidence on the inner side of the band runs approximately parallel to this updraft. The eyewall updraft is distinctly asymmetric, with stronger ascent on the side opposite to the band, near where the downwind extension of the updraft from the vorticity band spirals into the eyewall.

Vertical profiles of radial and azimuthal wind components are plotted at three points, $6 \mathrm{~km}$ inside of, and $6 \mathrm{~km}$ and $18 \mathrm{~km}$ outside of, the band axis, at the midpoint of the band. The profiles of azimuthal wind (Fig. 2i) display a marked wind maximum near 400-m height on the inside, and 600-800-m height on the outside, of the band. It is apparent that these maxima are within the inflow layer, and that the inflow layer similarly varies in depth across the band (Fig. 2h). The boundary layer flow in the vicinity of the band is thus very similar to that near a concentric ring of vorticity, and to the primary eyewall. It is also strongly reminiscent of the observed cross-band differences in Barnes and Powell (1995). Figures $2 \mathrm{~d}$ and $2 \mathrm{e}$ plot the jet factor (the strength of this wind maximum, relative to the balanced flow) and its height, showing that this region of relatively strong flow closely follows the band axis. To clearly distinguish such flow from the well-known supergradient flow in axisymmetric storms, we shall refer to it as super-NLB flow. The height of the maximum super-NLB winds decreases markedly across the band, similar to the supergradient jet near an eyewall. The spiral band is also collocated with a local maximum in the surface wind factor (SWF; the ratio of the $10-\mathrm{m}$ wind speed to the balanced wind speed), again similar to that found near an inner or outer eyewall (Fig. 2f). The higher values of SWF near the vorticity band are consistent with the recommendation by Franklin et al. (2003), based on their analysis of dropsonde data, of modestly higher SWF in convective areas of the storm.

The flow differences induced by the vorticity band are further analyzed in Fig. 3, which plots the differences between this simulation and a similar one with no band. The 10-m flow (Fig. 3a) is accelerated along and outside of the band, but weakened inside. These changes are of the same sign but lesser magnitude than those induced in the balanced flow by the vorticity band (Fig. 1). The near-surface streamlines of the flow difference show a marked confluence very close to the band axis (Fig. 3a). The band substantially increases the updraft along and predominantly near its axis, with a marked subsidence perturbation inside (Fig. 3b). Both of these features extend well downwind of the band's downwind end. The markedly different shapes of the wind profiles inside and outside of the band axis are confirmed by the change in wind turning: the turning of the wind with height is increased outside of the band, but markedly reduced on the inside, with the largest changes only $6 \mathrm{~km}$ inside of the band axis (Fig. 3c).

Figures $4 \mathrm{a}-\mathrm{c}$ show radius-height sections of the flow at an azimuth of $90^{\circ}$, the midpoint of the band. The two super-NLB azimuthal wind maxima, two inflow maxima, and two updrafts are clearly apparent, with one of each of these maxima near the RMW and the other near the spiral band axis. Figure $4 \mathrm{~d}$ shows a line plot of the balanced flow and its vorticity, the 10-m horizontal wind components, and the $1-\mathrm{km}$ vertical velocity along this radial. The updraft (magenta) at the rainband is clearly stronger than that at the eyewall, and is approximately $9 \mathrm{~km}$ inside of the balanced wind maximum (red), but less than $2 \mathrm{~km}$ outside of the local maximum in balanced vorticity (cyan). The flow here is strongly similar to that obtained with a concentric ring of enhanced vorticity, that is, to cyclones with an incipient or actual secondary wind maximum (Kepert 2013; Kepert and Nolan 2014; Zhang et al. 2017).

It is clear from Fig. 2 that the features in the flow, including the updraft, super-NLB flow, and enhanced inflow are relatively weak at the upwind end of the band and do not fully develop until farther downwind. It is also apparent that these features also extend some distance downwind from the downwind end of the band. The variation of the along-band flow is further examined in Fig. 5, which shows the location and strength of the updraft as a function of azimuth along the band. The updraft progressively grows to about a $90^{\circ}$ azimuth, and then weakens toward the end of the band. It is located outside the band axis at the upwind end, but approaches the axis by midband and then crosses to the inside. The discontinuity in position near the downwind end of the band is because the updraft has split, and this analysis chooses the updraft nearest the band axis. Figure 5 also shows (dashed curves) the updraft induced by a concentric ring of vorticity of the same strength and Gaussian structure, and at the same radius as the band axis is at each particular azimuth. $^{2}$ It is clear from this figure that the updraft strength at the upwind end of the band is much weaker than the corresponding concentric ring, is substantially stronger midband, and then weakens toward the downwind end. The behavior here is suggestive of the flow

\footnotetext{
${ }^{2}$ The simulations that these curves were derived from are similar to those in Kepert (2013), except that the vorticity structure here is prescribed as a Rankine vortex plus an annular Gaussian bump, rather than piecewise constant vorticity segments with a skirt. The rings at lower radii here induce a stronger updraft than those farther out because the radial vorticity gradient is the sum of the gradients due to the base vortex and the Gaussian band, and the gradient associated with the base vortex increases in magnitude inward sufficiently to dominate the decline of the $\left(\zeta_{\mathrm{gr}}+f\right)^{-2}$ term.
} 

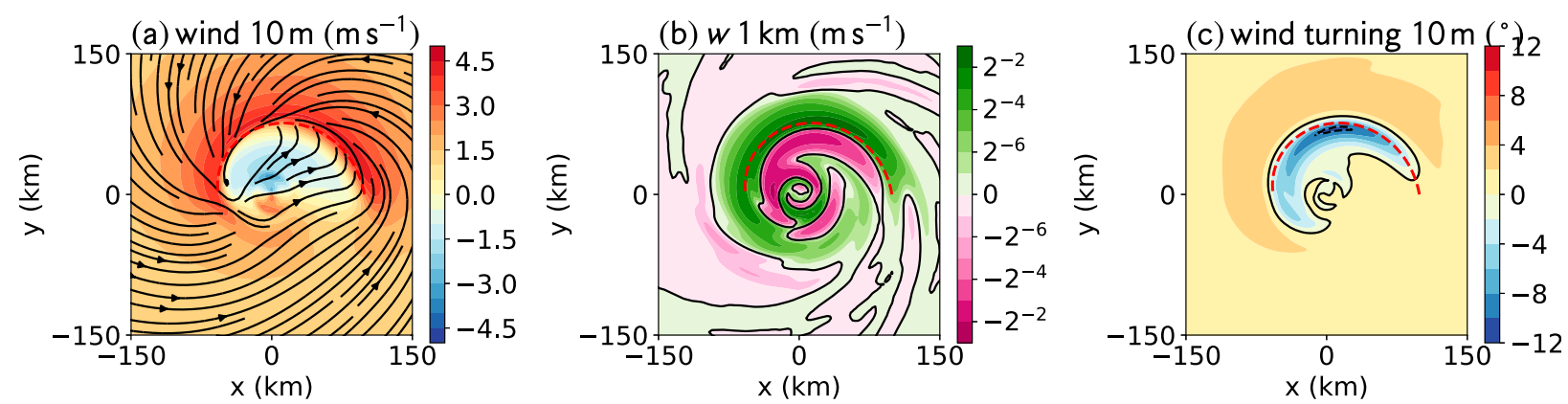

FIG. 3. Flow differences between simulations with and without a rainband. (a) Magnitude and streamlines of the vector 10-m wind difference, (b) the vertical velocity difference at $1.048 \mathrm{~km}$, and (c) differences in the wind turning. The wind turning is the difference in direction between the 10-m wind and the balanced flow and is positive if the $10-\mathrm{m}$ wind is directed more toward the vortex center. The red dashed curve marks the axis of the rainband vorticity. The with-rainband simulation is that shown in Fig. 2, while the without-rainband simulation has the same base vortex but no rainband.

adjusting to the presence of the band, overshooting an equilibrium then settling back, and will be analyzed in more detail in section 4 a.

\section{b. Sensitivities}

We now examine the sensitivity of the flow near the vorticity band to the band's strength, crossing angle, width, length, and radius.

If we alter the strength of the vorticity band, the strength of the boundary layer flow features change in approximate proportion. We show plots of the $1-\mathrm{km}$ updraft, the jet strength, and the surface wind factor for simulations with the band strength halved (Figs. 6a-c) and doubled (Figs. 6d-f) compared to the base case. While the magnitude has changed, the spatial distribution of these features is less affected, with the updraft still crossing from outside to inside of the band in similar fashion in these simulations. The eyewall updraft becomes progressively less symmetric with stronger spiral bands, but this result is at least partly due to the reduced balanced wind speed on the side of the eyewall toward the band in the strong-band simulations. Figures $7 \mathrm{a}$ and $7 \mathrm{~b}$ show the perturbation updraft strength, measured at the updraft axis, as a function of azimuth for a wider range of band strengths, confirming that this quantity is approximately linear with band strength at the upwind part of the band. ${ }^{3}$ This near proportionality is broken toward the downwind end, where the azimuth of maximum updraft decreases for stronger bands. Band strength has only a modest effect on the updraft location, with weaker bands tending to produce an updraft closer to the band axis.

\footnotetext{
${ }^{3}$ Note that the perturbation updraft (i.e., with the no-band simulation subtracted off) is plotted in Fig. 7, while the total fields are plotted in Fig. 6 .
}

Figure 8 shows the same three flow quantities as in Fig. 6 for the original band strength but with crossing angles of $0^{\circ}$ and $20^{\circ}$. We note first that the updraft becomes stronger and narrower with increased crossing angle, and that the subsidence immediately inside of the band likewise strengthens. The location of these features shifts outward with increased crossing angle, to the extent that the vorticity band's axis is largely in a region of frictional descent with a crossing angle of $20^{\circ}$. While the updraft becomes stronger with increased crossing angle, the strength of the super-NLB jet has the opposite trend, being strongest (of the cases shown) for the semicircular band. This impression is confirmed in Figs. 7c and 7d, which shows the peak 1-km updraft strength increases steadily with band-crossing angle, while the updraft is also displaced steadily farther out from the band axis for the larger crossing angles. Indeed, for these latter cases, the band axis is typically closer to the downdraft than to the updraft. The surface inflow angle in the simulation with no rainband is about $20^{\circ}$ at the radii of the rainbands, while the inflow angle averaged up to $1-\mathrm{km}$ height is about $13^{\circ}$. As the bandcrossing angle increases, progressively more of the boundary layer flow would become directed from inside the band to outside unless the band itself modifies the inflow. However, the inflow near the band does increase with greater crossing angle, such that the structure and magnitude of the inflow on either side of the band vary little with crossing angle.

Figure 9 shows the flow for narrower and wider bands, but the same amplitude. Halving the width reduces the updraft width by a lesser proportion, but has relatively little effect on the updraft strength. In the wider band, the updraft is broader and peaks outside of the axis of the vorticity band, but the peak updraft is similar to the base case, while the downdraft inside of the band is greatly reduced. The super-NLB flow increases in 


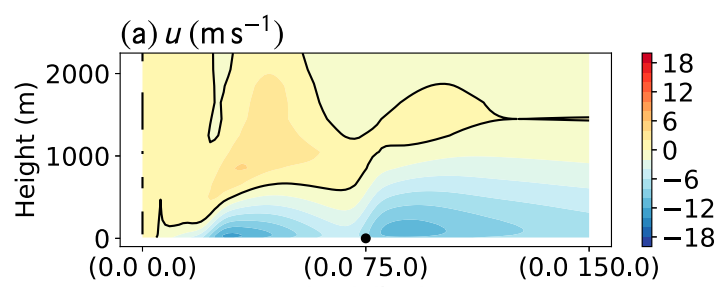

(b) $v\left(\mathrm{~m} \mathrm{~s}^{-1}\right)$

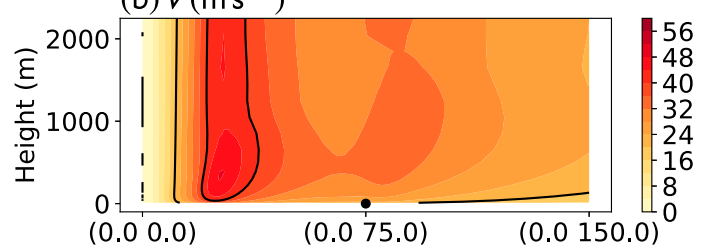

$(\mathrm{x}, \mathrm{y})(\mathrm{km})$

(c) $w\left(\mathrm{~m} \mathrm{~s}^{-1}\right)$

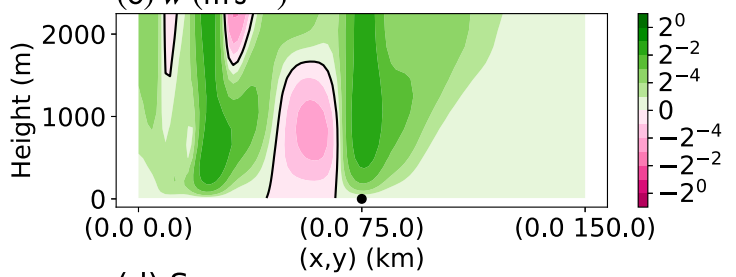

(d) Summary

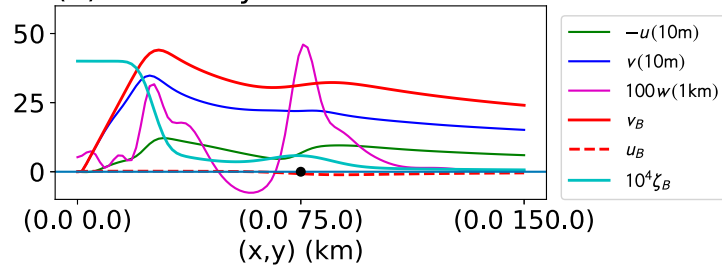

FIG. 4. Radius-height sections along the radial line through the midpoint of the spiral vorticity band, for the simulation shown in Fig. 2, of (a) radial velocity with the zero contour shown in black, (b) azimuthal velocity, and (c) vertical velocity with the zero contour shown in black. (d) Summary of the flow along the same radial, showing the radial (red dashed) and the azimuthal (red solid) balanced flow along with its vorticity (cyan; multiplied by $10^{4}$ ), the 10-m inflow (green), the 10-m azimuthal flow (blue), and the 1-km vertical velocity (magenta; multiplied by 100).

strength with bandwidth, and peaks outside of the band axis for the wider band. Figures $7 \mathrm{e}$ and $7 \mathrm{f}$ shows that the updraft strength has a nonmonotonic relationship with bandwidth scale, but that the updraft is steadily positioned farther outward for wider bands.

Shortening the band (Figs. 10a-c) has the expected effect that the response also becomes shorter. It still, however, extends beyond the downwind end of the band, with a residual updraft spiraling in to the eyewall. Perhaps the most interesting of these cases is the zero-length band, just an isolated vorticity blob, which induces a band of ascent spiraling into the eyewall. Remarkably, the resulting updraft increases steadily downwind from the vorticity blob, until it spirals into the eyewall updraft (Figs. 10d-f).

\section{(a) Updraft strength}

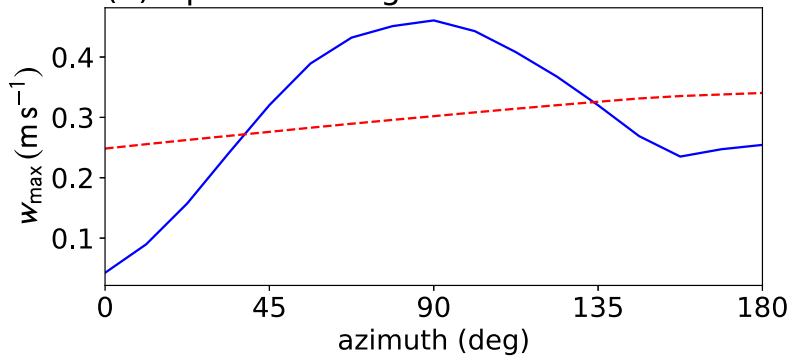

(b) Updraft location

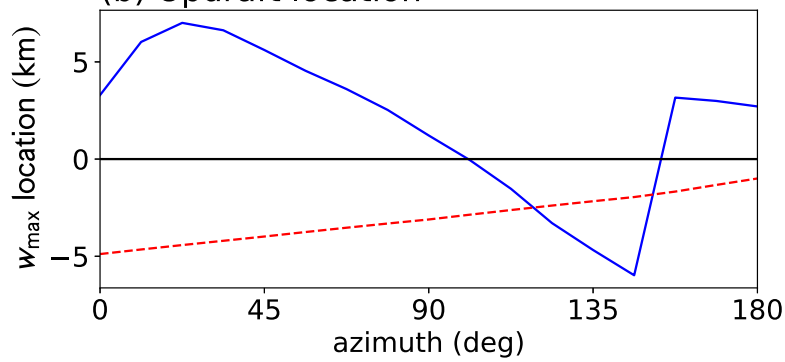

FIG. 5. Along-band variation of the frictional updraft. (a) Updraft strength at $1.048 \mathrm{~km}$ and (b) displacement of the updraft axis from the vorticity band axis (positive values indicate outward displacement). The solid blue curves are for the spiral band, and the dashed red curves are for concentric rings at the same radius as the spiral band at that azimuth.

Finally, we look at the effect of varying the radial location of the band. Here, the effects on the updraft are remarkably small. Figure 11 shows plots for bands with central radii of 50 and $150 \mathrm{~km}$. The subsidence on the inside of the band is stronger, and the updraft is closer to the axis for the larger-radius band, but there is little impact on the strength of the updraft. The super-NLB jet is much more marked when the band is at larger radius, and the SWF is also greater. Similar differences are obtained with concentric-ring vorticity perturbations at these radii (not shown). Figures $7 \mathrm{~g}$ and $7 \mathrm{~h}$ confirm the small effect on updraft strength, with a tendency for the peak updraft to be located closer to the upstream end for bands located at larger radii. The displacement of the updraft maximum from the band axis shows a similar shape in all cases, but the displacement is greater for larger-radius bands.

We also simulated some cases with multiple vorticity bands. In these cases, each band produced a flow perturbation similar to that for a single band, so the results are not shown.

\section{c. Intermittent bands}

We have modeled the vorticity bands as continuous arcs of logarithmic spirals, and argued that the vortex stretching in rainband convection is their source. However, convection is not evenly distributed along a rainband, and 


\section{Sensitivity to band strength}
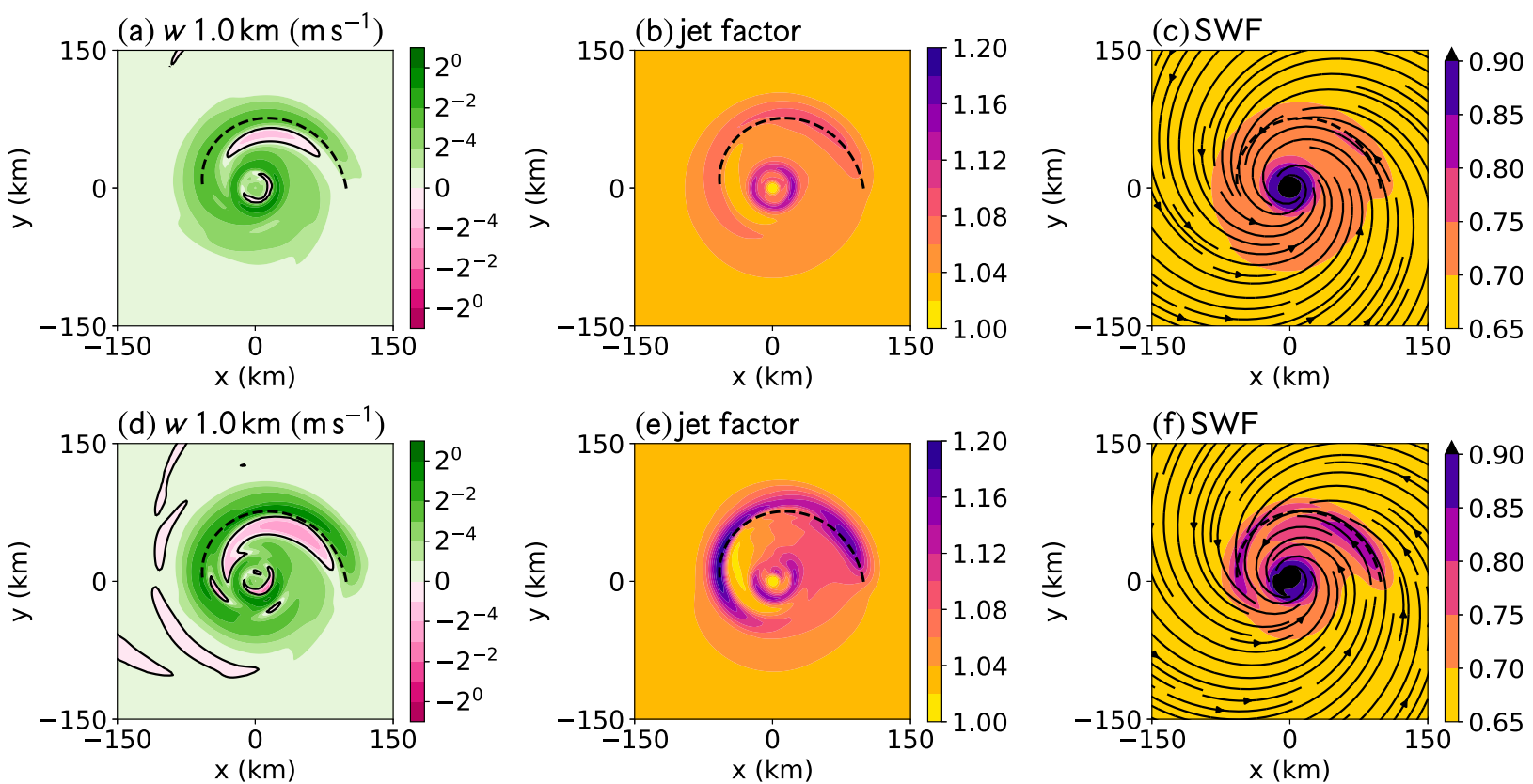

FIG. 6. The sensitivity to vorticity band strength. (a) The vertical velocity at $1 \mathrm{~km}$, (b) the maximum wind speed in the column divided by the balanced wind speed, and (c) the surface wind factor, for a vorticity band that has half the amplitude of that shown in Figs. 2 and 4 . (d)-(f) As in (a)-(c), but the vorticity band has twice the amplitude of that in Figs. 2 and 4.

in reality rainbands have a complex, multiscale alongband structure (Willoughby 1988; May 1996; Houze 2010; Didlake and Houze 2009, 2013a,b). In response to this, we performed several simulations with intermittent, rather than continuous, bands. Figure 12 shows the flow from one of these, which was similar to that in Fig. 2 except that the band is broken into five Gaussian hills, evenly spaced in the azimuth along the band axis, and with the amplitude increased so that the total rainband vorticity is unchanged. This patchiness is perhaps closer to real rainbands. The spacing of these hills is wide enough that flow perturbations caused by each hill are clearly apparent. These hillscale perturbations are embedded in a larger spiral-band flow structure much like that found with the continuous band. Given that the boundary layer flow perturbation extends downwind of a single Gaussian hill (Figs. 10d-f), it is perhaps not surprising that the effect of several such blobs can combine to produce a flow similar to that in a continuous band.

\section{Discussion}

\section{a. Frictional adjustment and nonlinear dynamics}

Regardless of the location, shape, and strength of the vorticity band, the updrafts nearly all begin with low amplitude at the upwind end and strengthen along the band before reaching a maximum, after which they typically decline. The updraft mostly begins on the outside of the band, before crossing to the inside farther along. These properties are most clearly apparent in Fig. 7. The steady increase in the updraft along the upstream part of the band suggests that the flow is gradually adjusting to the changed conditions as it encounters the band. Eliassen and Lystad (1977) proposed that the adjustment time scale for air columns in the tropical cyclone boundary layer is $I^{-1}$, where $I$ is the inertial stability, and this time scale was confirmed by the spinup experiments of Kepert (2017). Kepert (2017) further noted that the linear and nonlinear boundary layer models have systematic differences in the radial location of the updraft in axisymmetric storms: while the former has the peak updraft close to the radius of maximum vorticity gradient, in the latter the updraft is displaced inward by a distance that scales as $-u_{10} / I$. The fact that the updrafts we have calculated for vorticity bands are outside the band axis at the upwind end, then approach and cross the band axis farther downwind, is therefore also consistent with this adjustment process.

The experiments of Kepert (2017) were for axisymmetric vortices, so the appropriate horizontal advection velocity was the radial velocity. In contrast, the present study involves asymmetric rainbands, so the azimuthal velocity likely dominates. However, spiral 
(a) Updraft strength

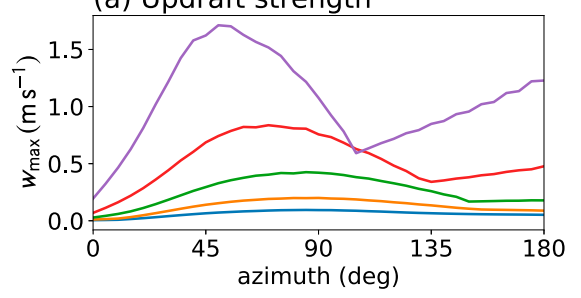

(c) Updraft strength

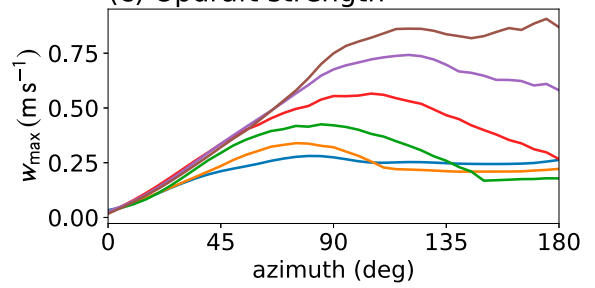

(e) Updraft strength

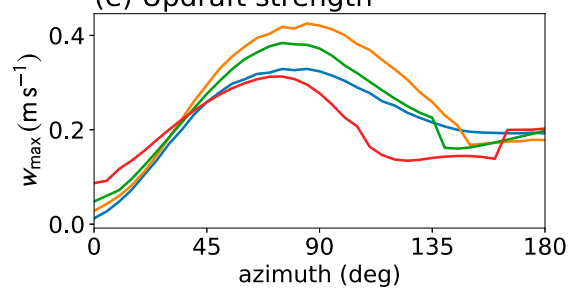

(g) Updraft strength

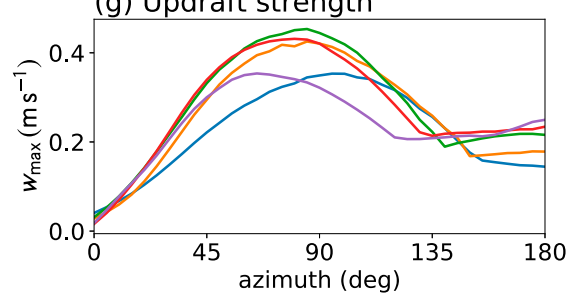

(b) Updraft location

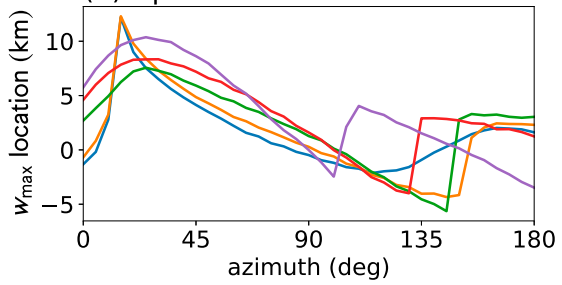

(d) Updraft location

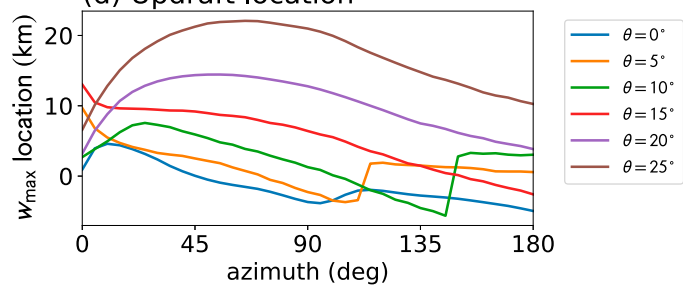

(f) Updraft location

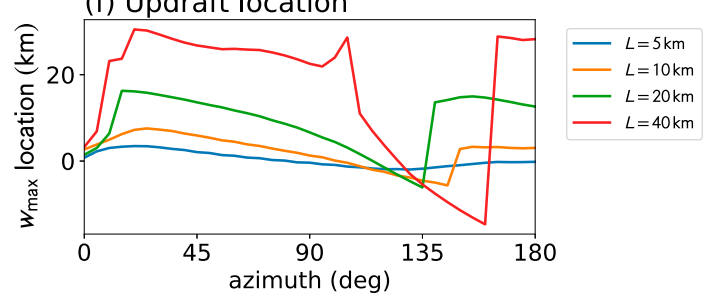

(h) Updraft location

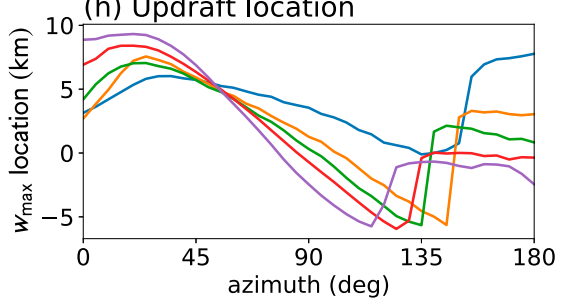

$\zeta_{0}=10^{-4} \mathrm{~s}^{-1}$

$\zeta_{0}=2 \times 10^{-4} \mathrm{~s}^{-1}$

$\zeta_{0}=4 \times 10^{-4} \mathrm{~s}^{-1}$

$\zeta_{0}=8 \times 10^{-4} \mathrm{~s}^{-1}$

$\zeta_{0}=1.6 \times 10^{-3} \mathrm{~s}^{-1}$

FIG. 7. Perturbation updraft (a) strength and (b) location as a function of azimuth for various band amplitudes as indicated in the legend. (c),(d) As in (a) and (b), respectively, but for band crossing angle. (e),(f) As in (a) and (b), respectively, but for bandwidth. (g),(h) As in (a) and (b), respectively, but for band midpoint radius.

rainbands change radius, and hence inertial stability, along their length, complicating the analysis. Hence, we now consider a series of experiments where the vorticity bands are semicircular rings.

Figures $13 \mathrm{a}$ and $13 \mathrm{~b}$ show the updraft strength and location, as a function of azimuth, for three such semicircular bands. ${ }^{4}$ The updraft has the same essential properties as spiral bands, in that it is weak at the upstream end and increases to a peak before settling back to a slightly lower value. It also begins outside of the vorticity band axis, then crosses to the inside of it. The

\footnotetext{
${ }^{4}$ The position of the along-band updraft maximum is somewhat influenced by the orientation of the band relative to the numerical grid. To reduce this influence, this figure shows the average of pairs of simulations oriented $45^{\circ}$ apart.
}

updraft finishes farthest inside for the largest-radius bands, similar to the situation with concentric rings (Kepert 2017). Figure 13c plots the azimuth of maximum updraft as a function of $V / r I$, where this quantity is evaluated for the cyclone without a rainband. There is a clear relationship between $V / r I$ and the development of the updraft, which is very nearly linear apart from a small discontinuity between half rings of 112- and $125-\mathrm{km}$ radius.

\section{b. Relationship to secondary eyewalls}

We have seen that, in many ways, the boundary layer's response to a spiral vorticity band is similar to its response to a concentric ring. It is tempting, therefore, to propose that a positive feedback exists in tropical cyclone rainbands similar to that proposed by Kepert (2013) for secondary eyewalls. In this feedback, buoyant 
Sensitivity to crossing angle
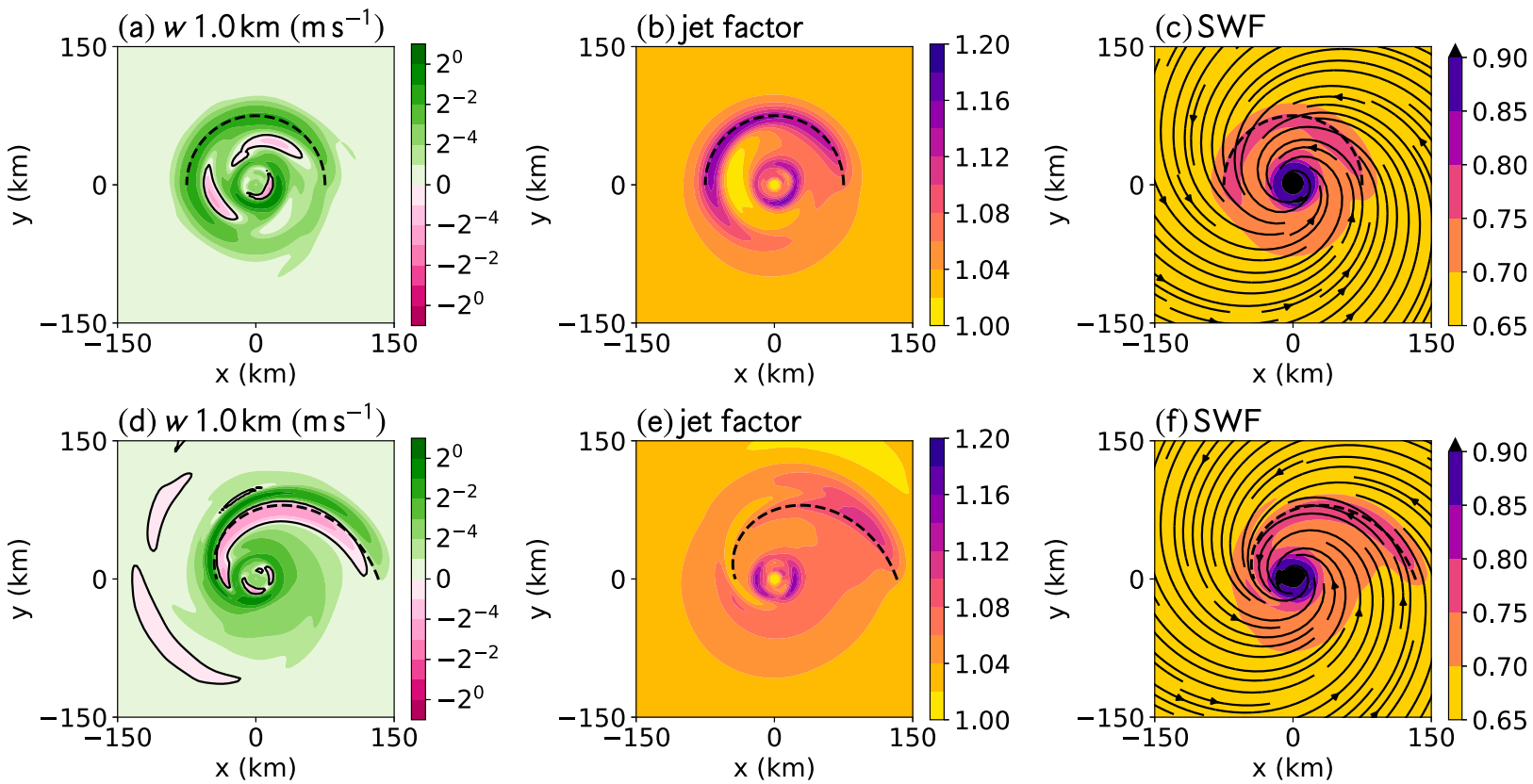

FIG. 8. The sensitivity to the vorticity band crossing angle. As in Fig. 6, but for (a)-(c) a band with a crossing angle of $0^{\circ}$ (i.e., a semicircle) and (d)-(f) a band with a crossing angle of $20^{\circ}$.

stretching of vortex tubes increases the vorticity, while the increased vorticity leads to increased frictional convergence, which in turn favors convection. However, some of the differences between spiral bands and concentric rings mean that this feedback may operate somewhat differently in spiral bands.

For the feedback to operate well, the frictional updraft must be located close to the maximum vorticity

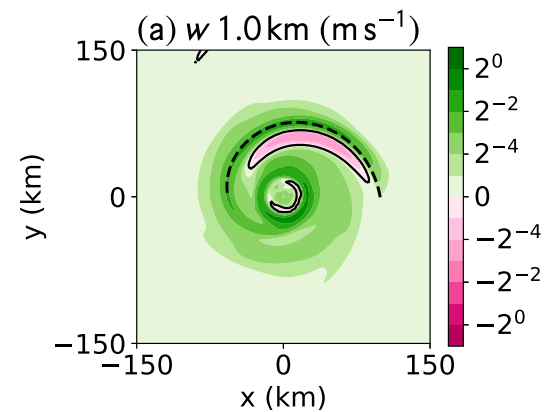

Sensitivity to band width
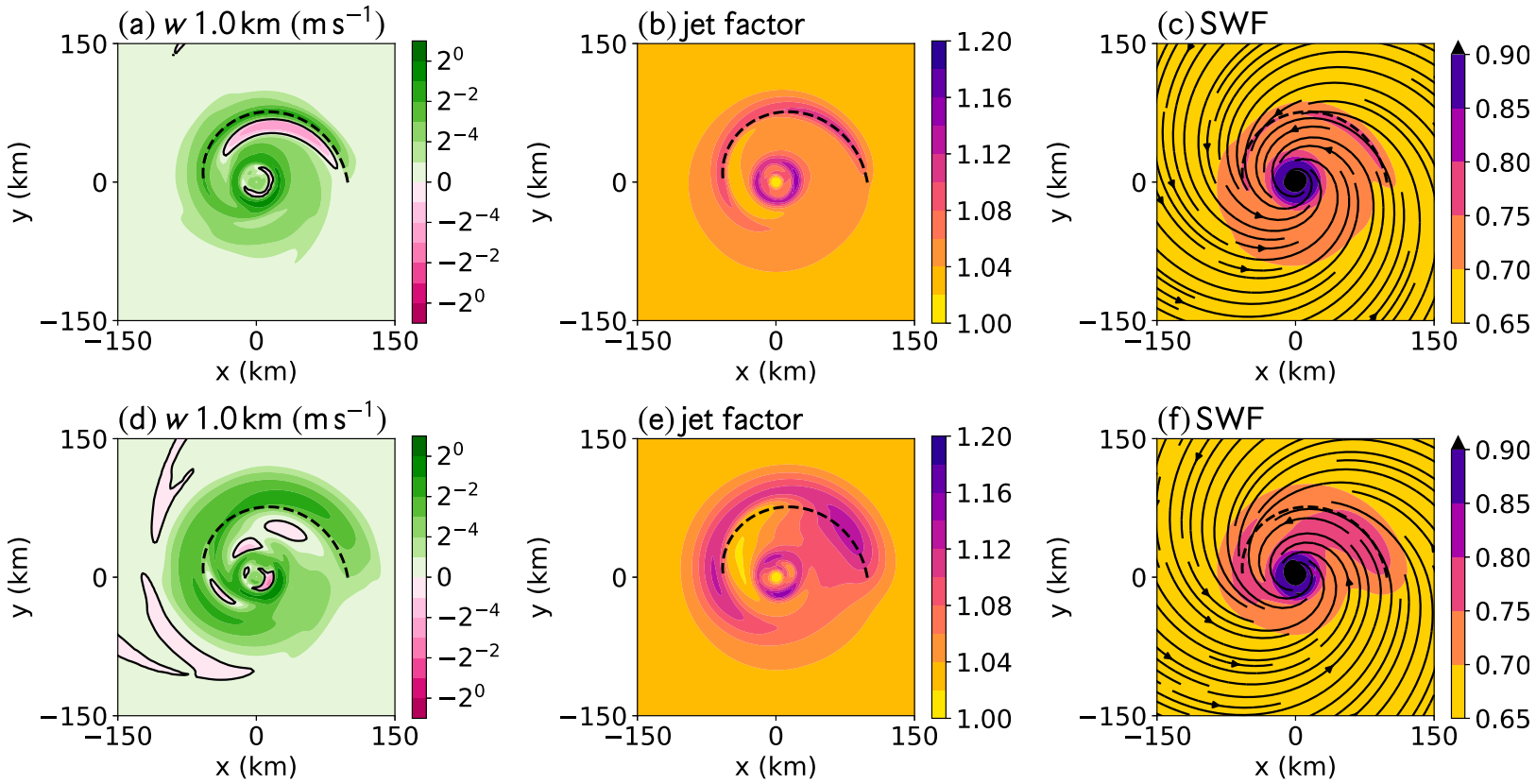

FIG. 9. The sensitivity to vorticity band scale width. As in Fig. 6, but for (a)-(c) a band with the scale width reduced to $5 \mathrm{~km}$ and (d)-(f) a band with the scale width increased to $20 \mathrm{~km}$. 


\section{Sensitivity to band length}
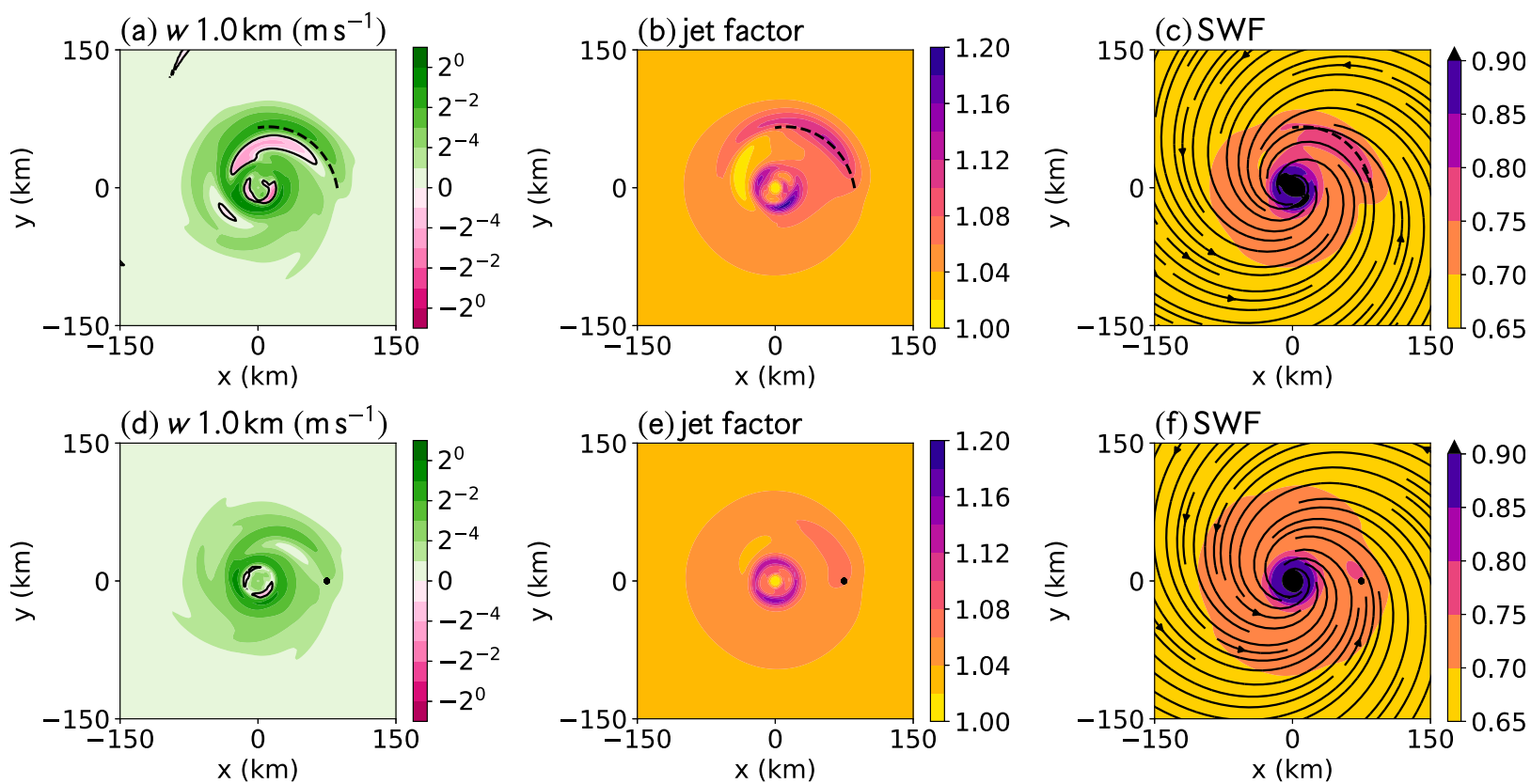

FIG. 10. The sensitivity to vorticity band length. As in Fig. 6, but for (a)-(c) a band with an azimuthal extent of $90^{\circ}$ and (d)-(f) a band with an azimuthal extent of $0^{\circ}$ (i.e., a single Gaussian vorticity perturbation).

perturbation; otherwise, the perturbation will tend to propagate rather than amplify (Kepert 2017). However, in most of the spiral band cases presented here, the updraft is outside of the band axis at the upwind end, and often crosses the axis to be inside of it at the downwind end (Figs. 7b,d,f,h). Thus, the proposed feedback will tend to increase the crossing angle of the band and, hence, act contrary to axisymmetrization.

\section{Sensitivity to band radius}
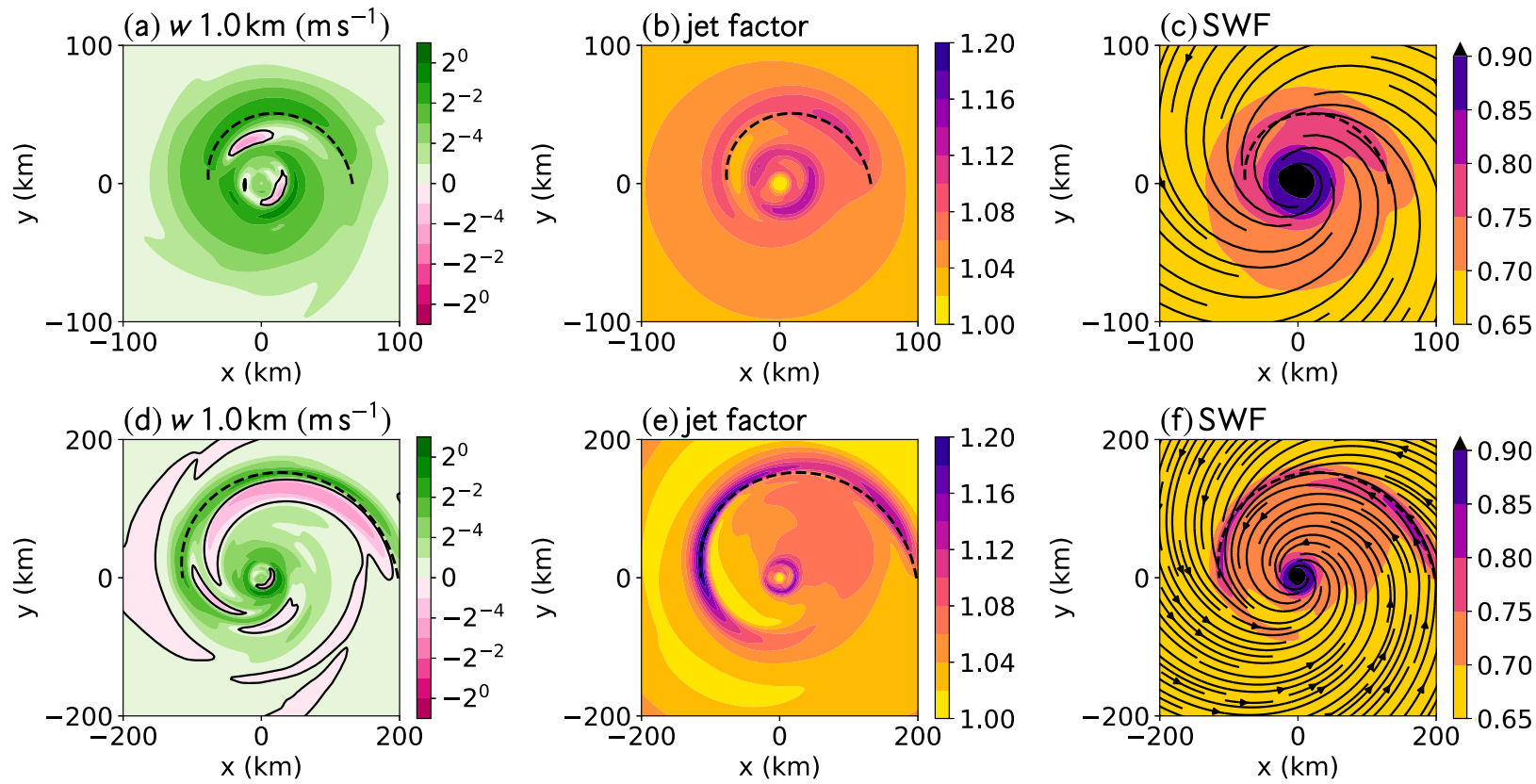

FIG. 11. The effects of vorticity band radial location. As in Fig. 6, but for (a)-(c) a band with a midpoint radius of $50 \mathrm{~km}$ and (d)-(f) a band with a midpoint radius of $150 \mathrm{~km}$. Note that the area plotted is reduced for (a)-(c) and increased for (d)-(f). 

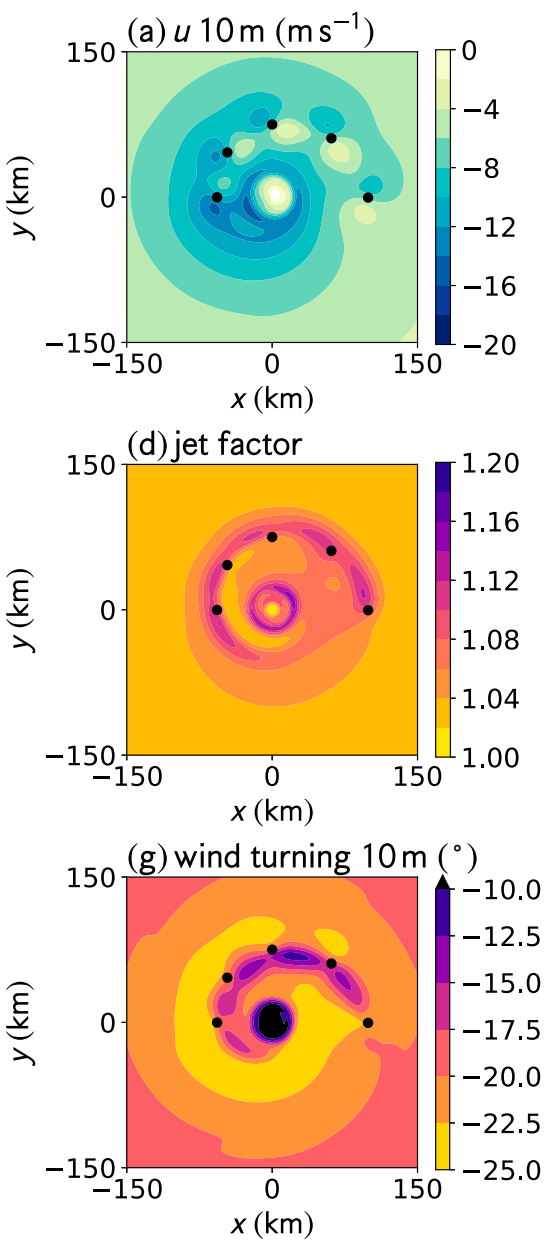

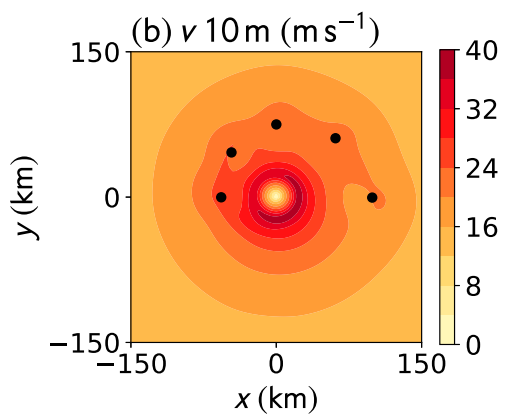

(e) jet height $(\mathrm{m})$
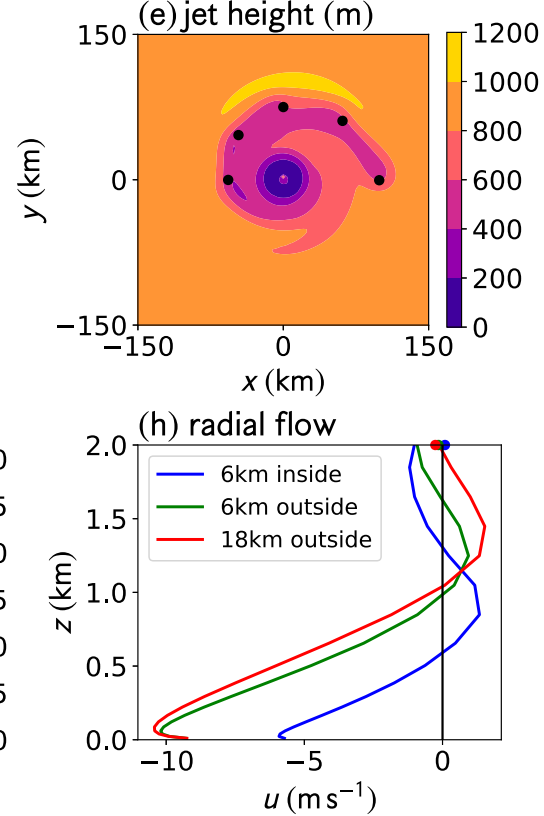
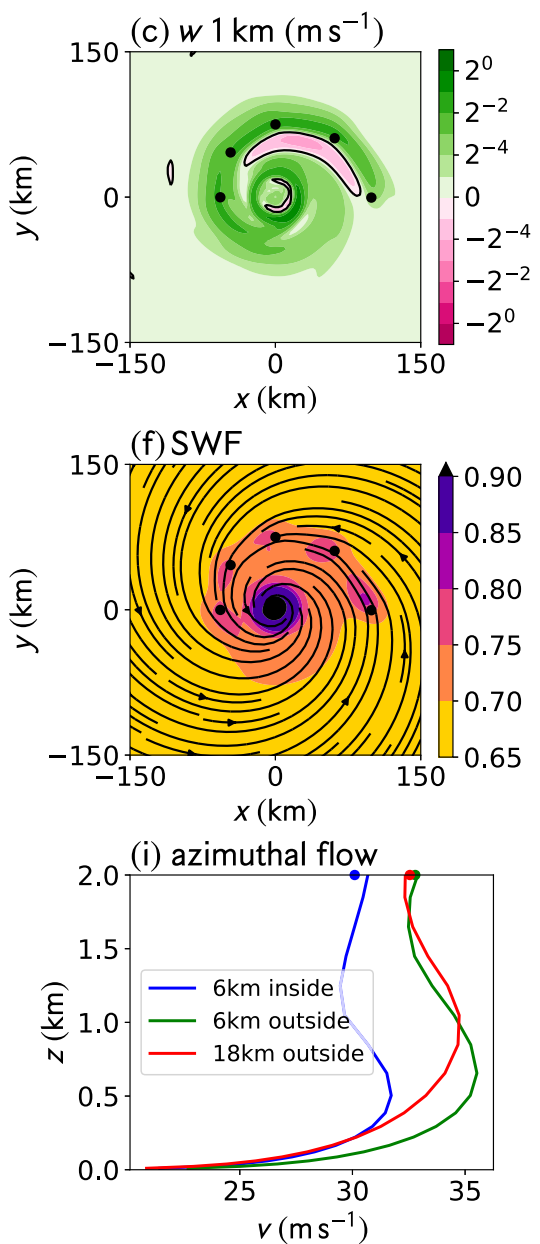

FIG. 12. As in Fig. 2, but for five individual Gaussian bumps of vorticity arranged along a spiral and indicated by the black dots in (a)-(g).

In addition, the feedback will be weaker at the upwind end of the band, where the updraft is establishing itself (Figs. 7a,c,e,g). Many cases also showed a decrease in the updraft strength toward the downwind end of the band, implying a weaker feedback there.

Observed rainbands tend to become less convective at the downwind end. This observation is perhaps a reason to doubt the proposed feedback, since our results show that the frictional updraft forcing extends beyond the end of the vorticity band and should therefore favor downwind growth of the band. However, there are other factors that could counter the influence of the updraft forcing, which in any case is getting weaker at the downwind end of the band. In particular, the downwind end of the band is in a region of stronger filamentation (Rozoff et al. 2006) and may also be experiencing stronger subsidence from the thermally forced part of the eyewall secondary circulation. Further, downdrafts from the convective cells in the upwind parts of the band are expected to reduce the boundary layer $\theta_{e}$, and this low-energy air may be advected downwind along the band and make it harder for lifting at the downwind end to create convection.

It is interesting to note that these negative factors will likely diminish if the rainband wraps up into a secondary eyewall. With the low-level flow aligned more across the band, downdraft air will be advected inward and away, and along-band differences in filamentation or larger-scale subsidence will diminish as the band axisymmetrizes.

The case of vorticity bands with large crossing angles is also interesting, for then the frictional updraft falls outside of the band axis. Under the hypothesized feedback, such bands would tend to migrate outward. In contrast, this feedback causes axisymmetric rings to migrate inward, more quickly when they are at large radius, tending to slower inward migration and more intensification as they move into regions of higher inertial stability (Kepert 2017). Hence, the preferred direction of movement under the feedback changes from outward to inward as the band axisymmetrizes. 
(a) Updraft strength

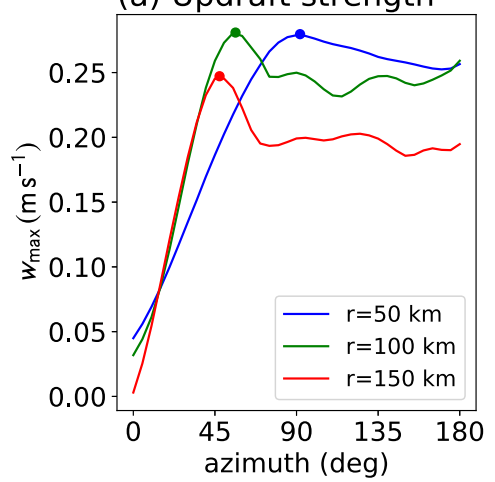

(b) Updraft location

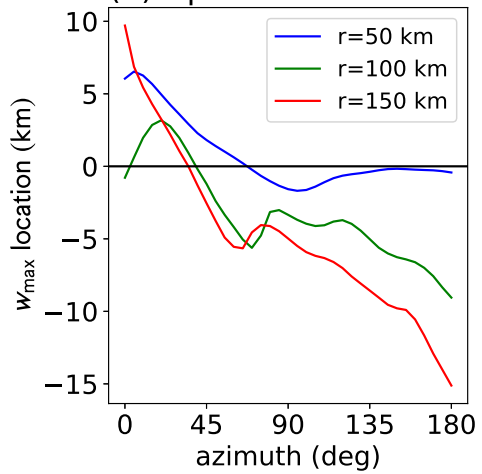

(c) Azimuth of max updraft

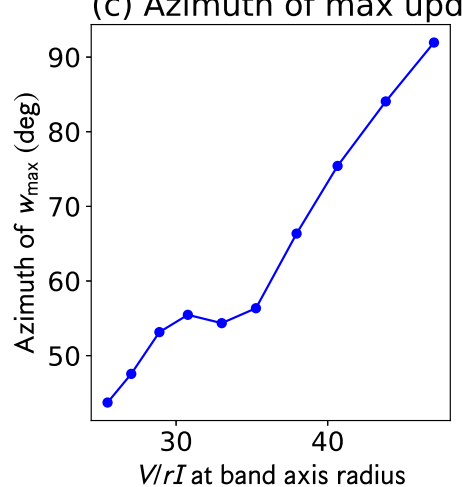

FIG. 13. Along-band variation of the perturbation frictional updraft for semicircular rings. (a) Strength of the band's updraft at 1.048 -km height as a function of azimuthal distance from the upstream end of the band, for bands at radii of 50,100, and $150 \mathrm{~km}$. The points show the azimuth of the maximum updraft, and the symmetric vortex updraft has been removed. (b) Radial displacement of the updraft axis from that of the vorticity band axis for the same cases as in (a). Positive values indicate outward displacement. (c) Azimuth of the maximum updraft within the semicircle, plotted against $V /(r I)$, for semicircle radii ranging from 50 to $162 \mathrm{~km}$. The smaller radii semicircles have larger $V /(r I)$.

The along-band wind maximum also becomes more prominent as the band axisymmetrizes, for it was in the cases with large crossing angle that the super-NLB flow was least prominent.

\section{Conclusions}

In this study, we have calculated the three-dimensional boundary layer flow associated with tropical cyclone rainbands, using a diagnostic model of the tropical cyclone boundary layer. The diagnostic model solves the horizontal momentum equations and continuity equation, given parameterizations of surface drag and turbulent momentum transport, in response to an imposed pressure field that represents the dynamical effects on the boundary layer of the rest of the cyclone. Moist processes, and hence that part of the secondary circulation induced by latent heat release, are neglected. Observations and theory suggest that rainbands are collocated with vorticity perturbations in the lower troposphere. We therefore represent rainbands in the model by spiral vorticity bands, and assume that the resulting flow satisfies the nonlinear balance equation. These two assumptions define the pressure field that forces the boundary layer model. In contrast to all previous studies with diagnostic models of the tropical cyclone boundary layer, the imposed pressure field is not axisymmetric.

This framework is able to reproduce many of the observed features in the boundary layer flow near rainbands. The inflow layer is deeper on the outside of the band than on the inside, and there is a marked alongband wind maximum, or low-level jet, in the upper boundary layer. We show that the wind speed in this jet is up to about $20 \%$ stronger than the local nonlinear balanced flow. The surface flow turns farther toward low pressure on the outside of the band than on the inside, and there is a region of marked low-level convergence near the band, leading to a frictional updraft comparable in magnitude to the eyewall updraft. There is also frictionally induced descent inward of this updraft. Finally, the surface wind factor, that is, the ratio of the $10-\mathrm{m}$ wind speed to the nonlinear balanced wind speed, is locally increased near the band. All of these characteristics extend a substantial distance downstream from the downwind end of the vorticity band, and do not develop their full amplitude until some distance downstream of the upwind end of the band.

We emphasize that these features are induced entirely by dry dynamical processes. Although one might expect momentum transports in cumulus downdrafts to lead to locally stronger surface winds near a tropical cyclone rainband, that process is not responsible for the increased surface wind factor found here, for the model does not include any cumulus processes. Similarly, the change in inflow depth and strength across the band here cannot be due to buoyant updrafts "consuming" the inflow. In the real atmosphere, of course, it is possible that moist processes further increase the phenomena we have noted here.

The above flow features are strongly similar to the flow near an outer annular vorticity perturbation, taken to represent an actual or incipient secondary eyewall by Kepert (2013) and Kepert and Nolan (2014). We therefore conclude that the boundary layer dynamics of spiral rainbands forms a continuum with the boundary layer dynamics of secondary eyewalls. Secondary eyewalls are typically observed to form via the "wrapping up" of a principal rainband; our results show that this evolution can occur without major disruption to their boundary layer dynamics. 
Kepert (2013) proposed that there is a positive feedback between lower-tropospheric vorticity and convection that contributes to secondary eyewall formation, whereby an annular vorticity perturbation in a tropical cyclone induces frictional convergence, that favors convection in that region, and that convection subsequently strengthens the vorticity perturbation through vortex stretching. Kepert and Nolan (2014) and Zhang et al. (2017) subsequently produced evidence that vorticity-induced boundary layer convergence is strongly influencing the location and strength of convection in separate WRF simulations. Clearly, a similar feedback could operate in spiral rainbands. However, there are aspects of spiral rainbands that are inconsistent with this feedback, so it cannot be the entirety of the dynamics. In particular, the frictional updraft is established progressively from the upstream end of the band, so any feedback must be weaker at that end. In addition, the updraft extends beyond the downwind end of the vorticity band, whereas in real rainbands, the downwind region is observed to be more stratiform, rather than convective, in character. However, as the present study neglects thermodynamics, it cannot account for factors such as cool moist downdraft air that could be advected toward the downwind end of the band and suppress convection there. Nor can it account for further strengthening of the surface convergence and updraft by buoyant convection.

The frictional updraft caused by a spiral band of vorticity is sensitive to the characteristics of the band. It is stronger for stronger bands, and also substantially stronger where the band-crossing angle is larger. It also increases, but to a lesser extent, when the band is at larger radius. Finally, it weakens for a wider band of the same amplitude. The updraft is typically located outside the band axis at the upwind end, but approaches and may cross to the inside of the band axis by the downwind end. When the updraft is sufficiently far outside of the vorticity band, for instance when the crossing angle is large, the axis of the band may actually fall in the area of frictional descent and may therefore be expected to be a region of suppressed convection. Based on these results, we suggest that an important factor in determining frictional ascent may be a negative radial gradient of vorticity, and that the actual updraft is displaced from this region by nonlinear processes similar to those elucidated for radial advection in concentric rings by Kepert (2017), but with the displacement here extended to include azimuthal advection. That hypothesized framework is consistent with the above sensitivities, as detailed in section 4 a.

This study is the first to use a diagnostic tropical cyclone boundary layer model with an asymmetric mass field. Although the asymmetric part of the mass field is small relative to the primary vortex, it can lead to frictional updrafts as strong as that at the eyewall. While we have here considered the case of mass asymmetries associated with rainbands, we will in future consider other sources of asymmetry, such as vertical wind shear, beta gyres, and the larger-scale environmental flow in which the storm is embedded.

\section{REFERENCES}

Abarca, S. F., and K. L. Corbosiero, 2011: Secondary eyewall formation in WRF simulations of Hurricanes Rita and Katrina (2005). Geophys. Res. Lett., 38, L07802, https://doi.org/ 10.1029/2011GL047015.

_, and M. T. Montgomery, 2013: Essential dynamics of secondary eyewall formation. J. Atmos. Sci., 70, 3216-3230, https://doi.org/10.1175/JAS-D-12-0318.1.

Barnes, G. M., and M. D. Powell, 1995: Evolution of the inflow boundary layer of Hurricane Gilbert (1988). Mon. Wea. Rev., 123, 2348-2368, https://doi.org/10.1175/1520-0493(1995) $123<2348$ :EOTIBL $>2.0$. CO; 2 .

_ - E. J. Zipser, D. Jorgensen, and F. D. Marks Jr., 1983: Mesoscale and convective structure of a hurricane rainband. J. Atmos. Sci., 40, 2125-2137, https://doi.org/10.1175/ 1520-0469(1983)040<2125:MACSOA > 2.0.CO;2.

Bell, M. M., M. T. Montgomery, and W.-C. Lee, 2012: An axisymmetric view of concentric eyewall evolution in Hurricane Rita (2005). J. Atmos. Sci., 69, 2414-2432, https://doi.org/ 10.1175/JAS-D-11-0167.1.

Chan, J. C. L., and J. D. Kepert, Eds., 2010: Global Perspectives on Tropical Cyclones: From Science to Mitigation. World Scientific Publishing, 444 pp.

Corbosiero, K. L., J. Molinari, and A. R. Aiyyer, 2006: The structure and evolution of Hurricane Elena (1985). Part II: Convective asymmetries and evidence for vortex-Rossby waves. Mon. Wea. Rev., 134, 3073-3091, https://doi.org/10.1175/ MWR3250.1.

Didlake, A. C., Jr., and R. A. Houze Jr., 2009: Convective-scale downdrafts in the principal rainband of Hurricane Katrina (2005). Mon. Wea. Rev., 137, 3269-3293, https://doi.org/ 10.1175/2009MWR2827.1.

$\longrightarrow$, and — 2013a: Convective-scale variations in the innercore rainbands of a tropical cyclone. J. Atmos. Sci., 70, 504523, https://doi.org/10.1175/JAS-D-12-0134.1.

- and $-2013 \mathrm{~b}$ : Dynamics of the stratiform sector of a tropical cyclone rainband. J. Atmos. Sci., 70, 1891-1911, https://doi.org/10.1175/JAS-D-12-0245.1.

Didlake, A. C., P. D. Reasor, R. F. Rogers, and W.-C. Lee, 2018: Dynamics of the transition from spiral rainbands to a secondary eyewall in Hurricane Earl (2010). J. Atmos. Sci., 75, 2909-2929, https://doi.org/10.1175/JAS-D-17-0348.1.

Eliassen, A., and M. Lystad, 1977: The Ekman layer of a circular vortex: A numerical and theoretical study. Geophys. Norv., 7, 1-16.

Emanuel, K. A., 1986: An air-sea interaction theory for tropical cyclones. Part I: Steady-state maintenance. J. Atmos. Sci., 43, 585-604, https://doi.org/10.1175/1520-0469(1986)043<0585: AASITF $>2.0 . \mathrm{CO} ; 2$.

Franklin, C. N., G. J. Holland, and P. T. May, 2006: Mechanisms for the generation of vorticity features in tropical cyclone 
rainbands. Mon. Wea. Rev., 134, 2649-2669, https://doi.org/ 10.1175/MWR3222.1.

Franklin, J. L., M. L. Black, and K. Valde, 2003: GPS dropwindsonde wind profiles in hurricanes and their operational implications. Wea. Forecasting, 18, 32-44, https://doi.org/10.1175/ 1520-0434(2003)018<0032:GDWPIH > 2.0.CO;2.

Houze, R. A., Jr., 2010: Clouds in tropical cyclones. Mon. Wea. Rev., 138, 293-344, https://doi.org/10.1175/2009MWR2989.1.

Huang, Y.-H., M. T. Montgomery, and C.-C. Wu, 2012: Concentric eyewall formation in Typhoon Sinlaku (2008). Part II: Axisymmetric dynamical processes. J. Atmos. Sci., 69, 662-674, https://doi.org/10.1175/JAS-D-11-0114.1.

Judt, F., and S. S. Chen, 2010: Convectively generated potential vorticity in rainbands and formation of the secondary eyewall in Hurricane Rita of 2005. J. Atmos. Sci., 67, 3581-3599, https://doi.org/10.1175/2010JAS3471.1.

Kepert, J. D., 2001: The dynamics of boundary layer jets within the tropical cyclone core. Part I: Linear theory. J. Atmos. Sci., 58, 2469-2484, https://doi.org/10.1175/1520-0469(2001)058<2469: TDOBLJ $>2.0 . \mathrm{CO} ; 2$.

- 2002a: Modelling the tropical cyclone boundary layer windfield at landfall. Modelling and Predicting Extreme Events: Extended Abstracts of Presentations at the Fourteenth Annual BMRC Modelling Workshop, A. J. Hollis and P. J. Meighen, Eds., Bureau of Meteorology Research Rep. 90, 81-84.

- 2002b: The impact of landfall on tropical cyclone boundary layer winds. 25th Conf. on Hurricanes and Tropical Meteorology, San Diego, CA, Amer. Meteor. Soc., 8A.1A, https:// ams.confex.com/ams/25HURR/techprogram/paper_37219.htm.

, 2006a: Observed boundary layer wind structure and balance in the hurricane core. Part I: Hurricane Georges. J. Atmos. Sci., 63, 2169-2193, https://doi.org/10.1175/JAS3745.1.

_ 2006b: Observed boundary layer wind structure and balance in the hurricane core. Part II: Hurricane Mitch. J. Atmos. Sci., 63, 2194-2211, https://doi.org/10.1175/JAS3746.1.

$\ldots$, 2010: Comparing slab and height-resolving models of the tropical cyclone boundary layer. Part I: Comparing the simulations. Quart. J. Roy. Meteor. Soc., 136, 1689-1699, https://doi.org/10.1002/qj.667.

_ 2012: Choosing a boundary layer parameterization for tropical cyclone modeling. Mon. Wea. Rev., 140, 1427-1445, https://doi.org/10.1175/MWR-D-11-00217.1.

- 2013: How does the boundary layer contribute to eyewall replacement cycles in axisymmetric tropical cyclones? J. Atmos. Sci., 70, 2808-2830, https://doi.org/10.1175/JAS-D-13-046.1.

_ 2017: Time and space scales in the tropical cyclone boundary layer, and the location of the eyewall updraft. J. Atmos. Sci., 74, 3305-3323, https://doi.org/10.1175/JAS-D-17-0077.1.

— within the tropical cyclone core. Part II: Nonlinear enhancement. J. Atmos. Sci., 58, 2485-2501, https://doi.org/10.1175/ 1520-0469(2001)058<2485:TDOBLJ > 2.0.CO;2.

— the boundary layer contribute to eyewall replacement cycles in axisymmetric tropical cyclones?"'. J. Atmos. Sci., 71, 46924704, https://doi.org/10.1175/JAS-D-14-0014.1.

- J. Schwendike, and H. Ramsay, 2016: Why is the tropical cyclone boundary layer not "well mixed"? J. Atmos. Sci., 73, 957-973, https://doi.org/10.1175/JAS-D-15-0216.1.

Li, Q., and Y. Wang, 2012: A comparison of inner and outer spiral rainbands in a numerically simulated tropical cyclone. Mon. Wea. Rev., 140, 2782-2805, https://doi.org/10.1175/MWR-D11-00237.1.
May, P. T., 1996: The organization of convection in the rainbands of Tropical Cyclone Laurence. Mon. Wea. Rev., 124, 807-815, https:// doi.org/10.1175/1520-0493(1996)124<0807:TOOCIT>2.0.CO;2.

_ , and G. J. Holland, 1999: The role of potential vorticity generation in tropical cyclone rainbands. J. Atmos. Sci., 56, 1224-1228, https:// doi.org/10.1175/1520-0469(1999)056<1224:TROPVG >2.0.CO;2.

_ J. D. Kepert, and T. D. Keenan, 2008: Polarimetric radar observations of the persistently asymmetric structure of Tropical Cyclone Ingrid. Mon. Wea. Rev., 136, 616-630, https://doi.org/10.1175/2007MWR2077.1.

Maynard, R. H., 1945: Radar and weather. J. Meteor., 2, 214 226, https://doi.org/10.1175/1520-0469(1945)002<0214: $\mathrm{RAW}>2.0 . \mathrm{CO} ; 2$.

Montgomery, M. T., and R. J. Kallenbach, 1997: A theory of vortex Rossby waves and its application to spiral bands and intensity changes in hurricanes. Quart. J. Roy. Meteor. Soc., 123, 435465, https://doi.org/10.1002/qj.49712353810.

Moon, Y., and D. S. Nolan, 2015a: Spiral rainbands in a numerical simulation of Hurricane Bill (2009). Part I: Structures and comparisons to observations. J. Atmos. Sci., 72, 164-190, https://doi.org/10.1175/JAS-D-14-0058.1.

$\longrightarrow$, and —_, 2015b: Spiral rainbands in a numerical simulation of Hurricane Bill (2009). Part II: Propagation of inner rainbands. J. Atmos. Sci., 72, 191-215, https://doi.org/10.1175/ JAS-D-14-0056.1.

Olson, L. N., and J. B. Schroder, 2018: PyAMG: Algebraic multigrid solvers in Python v4.0. Accessed 20 September 2018, https://pyamg.readthedocs.io/en/stable.

Ooyama, K. V., 1969: Numerical simulation of the life cycle of tropical cyclones. J. Atmos. Sci., 26, 3-40, https://doi.org/ 10.1175/1520-0469(1969)026<0003:NSOTLC >2.0.CO;2.

Powell, M. D., 1990: Boundary layer structure and dynamics in outer hurricane bands. Part I: Mesoscale rainfall and kinematic structure. Mon. Wea. Rev., 118, 891-917, https://doi.org/ 10.1175/1520-0493(1990)118<0891:BLSADI $>2.0 . C O ; 2$.

—_, E. W. Uhlhorn, and J. D. Kepert, 2009: Estimating maximum surface winds from hurricane reconnaissance measurements. Wea. Forecasting, 24, 868-883, https://doi.org/ 10.1175/2008WAF2007087.1.

Ramsay, H. A., L. M. Leslie, and J. D. Kepert, 2009: A highresolution simulation of asymmetries in severe Southern Hemisphere Tropical Cyclone Larry (2006). Mon. Wea. Rev., 137, 4171-4187, https://doi.org/10.1175/2009MWR2744.1.

Raymond, D. J., and M. J. Herman, 2012: Frictional convergence, atmospheric convection, and causality. Atmósfera, 25, 253-267.

Reasor, P. D., M. T. Montgomery, F. D. Marks Jr., and J. F. Gamache, 2000: Low-wavenumber structure and evolution of the hurricane inner core observed by airborne dual-Doppler radar. Mon. Wea. Rev., 128, 1653-1680, https://doi.org/10.1175/ 1520-0493(2000)128<1653:LWSAEO > 2.0.CO;2.

Rozoff, C. M., W. H. Schubert, and B. D. McNoldy, 2006: Rapid filamentation zones in intense tropical cyclones. J. Atmos. Sci., 63, 325-340, https://doi.org/10.1175/JAS3595.1.

Samsury, C. E., and E. J. Zipser, 1995: Secondary wind maxima in hurricanes: Airflow and relationship to rainbands. Mon. Wea. Rev., 123, 3502-3517, https://doi.org/10.1175/1520-0493(1995) $123<3502:$ SWMIHA $>2.0$.CO;2.

Schwendike, J., and J. D. Kepert, 2008: The boundary layer winds in Hurricanes Danielle (1998) and Isabel (2003). Mon. Wea. Rev., 136, 3168-3192, https://doi.org/10.1175/2007MWR2296.1. 
Shapiro, L. J., 1983: The asymmetric boundary layer flow under a translating hurricane. J. Atmos. Sci., 40, 1984-1998, https://doi.org/ 10.1175/1520-0469(1983)040<1984:TABLFU>2.0.CO;2.

Terwey, W. D., and M. T. Montgomery, 2008: Secondary eyewall formation in two idealized, full-physics modeled hurricanes. J. Geophys. Res., 113, D12112, https://doi.org/ 10.1029/2007JD008897.

Wexler, H., 1947: Structure of hurricanes as determined by radar. J. N. Y. Acad. Sci., 48, 821-845, https://doi.org/10.1111/ j.1749-6632.1947.tb38495.x.
Willoughby, H. E., 1988: The dynamics of the tropical cyclone core. Aust. Meteor. Mag., 36, 183-191.

Wu, C.-C., Y.-H. Huang, and G.-Y. Lien, 2012: Concentric eyewall formation in Typhoon Sinlaku (2008). Part I: Assimilation of T-PARC data based on the ensemble Kalman filter (EnKF). Mon. Wea. Rev., 140, 506-527, https://doi.org/ 10.1175/MWR-D-11-00057.1.

Zhang, F., D. Tao, Y. Q. Sun, and J. D. Kepert, 2017: Dynamics and predictability of secondary eyewall formation in sheared tropical cyclones. J. Adv. Model. Earth Syst., 9, 89-112, https:// doi.org/10.1002/2016MS000729. 